\title{
The role of phonotactic principles in language processing
}

\section{Christiane Ulbrich, Phillip M. Alday, Johannes Knaus, Paula Orzechowska \& Richard Wiese}

To cite this article: Christiane Ulbrich, Phillip M. Alday, Johannes Knaus, Paula Orzechowska \& Richard Wiese (2016) The role of phonotactic principles in language processing, Language, Cognition and Neuroscience, 31:5, 662-682, DOI: 10.1080/23273798.2015.1136427

To link to this article: http://dx.doi.org/10.1080/23273798.2015.1136427

舟 Published online: 24 Feb 2016.

Submit your article to this journal $₫$

Џ Article views: 427

Q View related articles $\longleftarrow$

View Crossmark data ¿

Citing articles: 1 View citing articles $\square$ 


\title{
The role of phonotactic principles in language processing
}

\author{
Christiane Ulbrich ${ }^{a}$, Phillip M. Alday ${ }^{b}$, Johannes Knaus ${ }^{c}$, Paula Orzechowska ${ }^{d}$ and Richard Wiese ${ }^{e}$

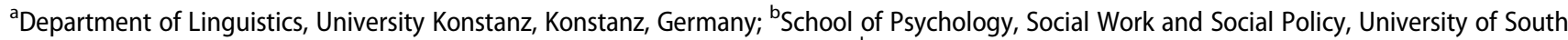 \\ Australia, Adelaide, Australia; ${ }^{C}$ Max Planck Digital Library, Munich, Germany; ${ }^{d}$ Faculty of English, Adam Mickiewicz University, Poznań, Poland; \\ eDepartment of German Linguistics, Philipps-Universität Marburg, Marburg, Germany
}

\section{ABSTRACT}

The paper reports the results of a learnability experiment with German speakers, investigating the role of universal phonotactic constraints and language use in language processing. Making use of an artificial language paradigm, participants learned nonce words with existent and non-existent German final consonant clusters adhering to or violating sonority sequencing principles postulated for consonant clusters. Behavioural data and event-related brain potentials in response to these cluster properties were obtained twice, before and after learning wordpicture-pairs. The results show (1) that learning and processing of final consonant clusters is facilitated by adherence to the sonority hierarchy, and (2) that actual existence of well- and illformed consonant clusters aids processing mechanisms. Thus, both implicit knowledge of universal phonotactic principles and frequency-based factors are demonstrated to play a role in the online-processing of words.

\section{ARTICLE HISTORY}

Received 15 October 2014

Accepted 16 December 2015

\section{KEYWORDS}

Sonority; learning; eventrelated potentials; phonological constraints; phonotactic

\section{Introduction}

Spoken language involves concatenating phoneme-like entities in order to form words and other units. For this concatenation of segments, languages have restrictions - it is obvious that not all logically possible combinations of segments found in a language are admitted. In phonological and psycholinguistic research, the nature of these restrictions thus is an important domain of theoretical and empirical study. The form of a meaningful unit usually consists of a particular sequence of phonological entities, and their perception and/or production involves the identification of this meaningful unit by means of its component sounds.

The distributions of sounds are governed by phonotactic rules and constraints, that is, phonotactic regularities defining what sound combinations may or may not be possible in a particular language. Phonotactic cues as well as prosodic cues are sub-lexical regularities used by listeners to identify word boundaries in the acoustic signal. Sub-lexical prosodic cues include pauses, temporal segmental variation such as final lengthening, intonation and metrical patterns (e.g. Shatzman \& McQueen, 2006a, 2006b). The most basic combination of sounds found is that of an alternating sequence of consonants and vowels, leading to CV-syllables, but combinations of adjacent consonants and vowels (i.e. consonantal/vocalic clusters) are possible, though in a limited way. The principles underlying this domain, called phonotactics, have been subject of study and debate, ranging from the work by Whitney (1865) and Sievers (1876/1901) to the recent survey by Parker (2012). Furthermore, the role of clusters and their underlying phonotactic constraints in the processing of words has been under scrutiny as well (Treiman, Fowler, Gross, Berch, \& Weatherston, 1995). Work in acquisition and processing has demonstrated a number of asymmetries: clusters are harder to acquire and use than simple forms, and structural and frequency-based explanations have been discussed for these asymmetries (Goad \& Rose, 2004; Kirk \& Demuth, 2005; Levelt, Schiller, \& Levelt, 2000; Munson, 2001). Furthermore, Nespor, Peña, and Mehler (2003) demonstrated that consonants play a prominent role (compared to vowels) for the identification of lexical items and for their acquisition. The notion of sonority has played a prominent role in the domain of structural principles governing phonotactics (see discussions by, e.g. Clements, 1990; Henke, Kaisse, \& Wright, 2012; Whitney, 1865). In contrast, recent models of phonology have stressed the role of use and experience. Specific evidence for the role of sonority was found by Treiman (1984) in behavioural experiments: in a syllable-forming task, participants grouped the vowel and the following consonant together, if the consonant was a liquid, but not (or to a lesser extent) if the consonant was some other, less sonorous, consonant. Using a lexical decision task, Levitt, Healy, and Fendrich (1991) confirmed these findings. 
Several attempts have been made in order to identify universal sub-lexical properties that are employed by infants in first language acquisition (e.g. Jusczyk, Luce, \& Charles-Luce, 1994; Kuhl, 2010; Mattys \& Jusczyk, 2001; Seidl, Cristià, Bernard, \& Onishi, 2009), and by adults in second language acquisition of natural and/or artificial languages in word segmentation (Chambers, Onishi, \& Fisher, 2010; Dell, Reed, Adams, \& Meyer, 2000; Endress \& Hauser, 2010; Goldrick \& Larson, 2008; Norris, McQueen, Cutler, \& Butterfield, 1997; Warker \& Dell, 2006). As some phonotactic constraints are seen as cross-linguistically similar, they are assumed to be part of universal linguistic, cognitive, articulatory, or auditory systems (e.g. Moreton, 2002). However, since languages differ considerably in their representation of phonotactic constraints, it seems indisputable that at least parts of this phonological knowledge have to be learned.

The present paper contributes to the debate about universal and languages-specific phonotactic constraints by examining the electrophysiological responses to two factors which determine the appearance of consonant clusters; namely sonority as well as their existence or non-existence in a particular language. The paper discusses these issues by looking at word-final consonant clusters of German and studying their role in the processing of nonce words.

In order to investigate the impact of implicit knowledge of certain phonological structures we conducted an experiment on word learning by native speakers of German. We adopted the learnability paradigm using an artificial grammar methodology. Artificial grammar learning (AGL) is commonly applied to study the role of universal primitives of language and the implicit knowledge thereof. We use the AGL paradigm to investigate the relative ease or difficulty with which consonant clusters can be learnt when violating or adhering to particular universal requirements. The universal primitive of interest in our case is wellformedness with respect to the Sonority Sequencing Principle (SSP). According to this principle, a syllable nucleus, often a vowel, constitutes the sonority peak in the centre of the syllable which is optionally preceded and followed by consonant(s) with sonority values (determined by a sonority hierarchy) decreasing progressively towards the syllable edges (e.g. Clements, 1990). The sonority hierarchy as well as the influence of actual existence of and exposure to certain phonological structures is in the focus of the experiment presented below.

Word learning was tested with the recognition of word-picture-pairs presented to the participants. Most of the existent literature concerned with phonotactic constrains is based on the unit of the syllable. However, if phonotactic constraints referred to the syllable level alone, speakers should not be sensitive to cross-syllabic information, and indeed some of the phonotactics of natural languages requires reference to other boundaries, such as word or morpheme edges. In Korean, for instance, $/ \mathrm{m} /$ is a permissible onset and $/ \mathrm{k} /$ a permissible coda, but only in word-initial and word-final position respectively. Thus, the fact that $/ \mathrm{k} . \mathrm{m} /$ sequences do not occur on word-medial syllable boundaries (e.g. Kabak \& Idsardi, 2007) cannot be accounted for at a syllableinternal level alone, although it requires reference both to the syllable sequence and to the sonority difference between $/ \mathrm{k} /$ and $/ \mathrm{m} /$. More evidence for word-level phonotactics comes from parsing experiments with infants acquiring English. In continuous speech, they identified words more rapidly edged with clusters that appear more frequently at word boundaries than those that appear more frequently at word-medial boundaries (Mattys, Jusczyk, Luce, \& Morgan, 1999).

Several alternative proposals for the domain of phonotactic constraints application have been made that do not require reference to the syllable level but rather draw on restrictions of the linear sequence of sounds and boundaries of words (e.g. Dziubalska-Kołaczyk, 2002; Steriade, 1999). These could explain contact restrictions as in the Korean example above, and would therefore solve the redundancy issue between word- and syllable-level constraints apparent in numerous languages.

Further support for the word-level as a domain of phonotactic constraints comes from sound-substitution experiments. Fowler (1993) reported that adults more readily replace word-initial onset consonants than consonants in word-medial onsets, that is, /v/ in vap.kem was easier to replace than $/ k /$. Similarly, applying a learning paradigm, Endress and Mehler (2010) recently found that adults learned restricted onset and coda consonant occurrence more easily when the restricted consonant was either in word-initial or word-final position as opposed to word-medial onset or coda position. They showed that, for instance, the restriction of " $\mathrm{f}$ " as onset and " $p$ " as coda in "fal.nip" was easier to learn than in "lap.fin" providing support for word-level representation in phonotactic learning.

\section{Learnability}

Previous research has shown that phonotactically legal and illegal words are learned (Bernard, 2011; Chambers et al., 2010) and processed differently (e.g. Bentin, Mouchetant-Rostaing, Giard, Echallier, \& Pernier, 1999). However, theoretical phonological approaches make different assumptions about the origin of those differences. In the framework of Universal Grammar (UG, 
Chomsky \& Halle, 1968), underlying universal principles regulate acquisition and resulting processing mechanism of language. In such an approach, the phonological system of a language users is based on abstract rules, constraints, or principles which are categorical and generalise over many cases. Categorisations and Generalisations over phonotactic regularities in this view are internal to language and derive, at least in part, from deep-rooted universal principles. In other words, individuals are equipped with an underlying knowledge about wellformedness of sound structures (e.g. groundbreaking work by Smith, 1973 or most papers in Kager, Pater, \& Zonneveld, 2004). On the contrary, more recent usage-based approaches understand language and its regulations as a dynamic adaptive system (Ellis \& Larsen-Freeman, 2006) that emerges on the basis of general cognitive functions and shapes through characteristics such as exposure and frequency in the input as well as usage (MacWhinney, 1999). In this view the representation of phonological phenomena is determined by the frequency of its use and receives a psycholinguistic interpretation reflected in the level of activation. Note, however, that it is logically possible that both views are correct to some extent: the actual use of language may be grounded in abstract principles as well as in usagebased frequency patterns.

Our experiment is designed to address hypotheses made on the basis of the two opposing theoretical approaches. On the one hand, we are interested in the processing of (il-)legal phonotactic constraints, namely sonority. On the other hand, we aim to show that not only the implicit knowledge of such a proposed phonological universal but also frequent exposure influences and in fact facilitates learning of structures that comply with the constraint. The learning paradigm has previously been successfully employed in several studies and in application of both real language tokens as well as nonor pseudo-words, the former referring to ill-formed and the latter to well-formed structures (Friederici, Bahlmann, Heim, Schubotz, \& Anwander, 2006). The generalisation of phonological constraints to unknown words provides important evidence for the existence of these constraints (e.g. Bernard, 2011; Chambers et al., 2010).

Studies on the neurolinguistic or psycholinguistic reality of phonological constraints are, however, limited. Rossi et al. (2011) investigated in a passive listening task the processing of pseudo-words designed according to the rules of German phonotactics vs. illegal nonce words. They found a larger N400 effect for phonotactically legal pseudo-words compared to the illegal nonce words, and interpreted the more pronounced effect as evidence for a stronger lexical activation in material adhering to phonotactic requirements. Similar event-related potential
(ERP) results were obtained by Friedrich and Friederici (2005). Twelve and 19 month olds as well as adults listened to pseudo-words, with phonotactically legal onsets, and nonce words, with phonotactically illegal onsets, as well as to real words. Simultaneously they were presented with pictures of known objects, either congruous or incongruous to the real words. Whilst in 12 month olds no effect was found for either congruous or incongruous or for pseudo- and nonce words, in 19 month olds and adults, incongruous words and pseudowords elicited an N400. This effect is generally interpreted as a reflection of lexical-semantic integration (Bentin et al., 1999; Holcomb, 1993; Holcomb \& Neville, 1990; Nobre \& McCarthy, 1994).

The results of an investigation of the Obligatory Contour Principle - a phonological constraint that prohibits the appearance of consecutive identical features in syllables, roots, and words (e.g. McCarthy, 1986; Odden, 1986) - in real words as well as in non- and pseudowords, revealed that the violation of the phonotactic constraint produces a Late Positive Component (LPC) in the electroencephalogram (EEG); Domahs, Kehrein, Knaus, Wiese, and Schlesewsky (2009). The LPC was interpreted as a result of a differentiation between licit and illicit neologisms and followed an earlier negativity (N400), which was ascribed to the detection of a nonexistent structure. A late positivity was previously found and associated with the re-analysis of syntactic complexity and ambiguity or the violation of (morpho-) syntactic structures (Friederici, 1995, 2002; Frisch \& Schlesewsky, 2005; Hagoort, 2003; Meerendonk, Chwilla, \& Kolk, 2013). The N400 component, however, has been ascribed to the differentiation between existent and non-existent words in numerous studies (Bentin et al., 1999; Holcomb, 1988, 1993; Holcomb \& Neville, 1990; Kutas \& Hillyard, 1980). Furthermore, there appears to be a differentiation in the $\mathrm{N} 400$ component in that only non-words with legal phonotactics elicit an early negativity, while illegal non-words do not, indicating sensitivity to the phonological structure of stimuli (Bentin et al., 1999).

In an functional magnetic resonance imaging (fMRI) investigation on English, Berent et al. (2014) studied differential brain responses in preferred and dis-preferred phonotactic structures with respect to adherence to the sonority hierarchy. The results, relevant for the present study since German and English can be assumed to exhibit similar (although not identical) connections to the sonority principle, revealed significant differences in the bold hemodynamic response to syllable structures violating or adhering to the sonority hierarchy. Both brain responses as well as behavioural results turned out to be gradient and mirrored the severity of violation, 
ranging from a large sonority rise on the one hand and a sonority fall on the other. The results showed that a small rise in sonority was deemed to be less ill-formed compared to a plateau, which in turn was still preferred over a fall in sonority (e.g. blif = large rise, bnif $=$ small rise, $b d i f=$ plateau, $l b i f=$ fall). Most relevant for the present study was the finding showing that a comparable differentiation of gradience in phonotactic violation was observed in the hemodynamic response at two sites, Broca's area (BA45) and its right hemisphere homologue.

The present study aims to shed light on the intricate interplay between phonotactic constraints and the existence of specific licit and illicit phonological structures. As shown in the discussed literature brain responses to phonotactic constraints and (non-)existence can attenuate or amplify each other or elicit specific patterns of processing mechanisms, that is, in EEG recordings the order of particular negativity or positivity effects. In order to show possible separate or integrated effects of the two phenomena, we chose a learnability paradigm.

\section{German phonotactics}

German is a language allowing reasonably complex consonantal clusters word-initially and word-finally. In wordfinal position, up to four consonants can be found, as in Herbst "autumn". Constraints for these combinations have been discussed in terms of cluster size (clusters of length two are more common than longer ones), place features (tongue-tip sounds are more common), and sonority (see below); see Wiese (1988) or Hall (1992) for a more detailed discussion. Explanations for the fact that some combinations exist, while many others do not, have been sought in terms of phonological markedness and/or perceptual preferences.

In any case, it is obvious that 20-25 consonantal phonemes in German (depending on the analysis of complex and/or marginal phonemes) yield only about 50-54 bisegmental word-final combinations (Wiese, 2000, ch. 7.5.2), with the exact number depending on the treatment of marginal clusters found in loan words. That is, the right word-edge can be marked by complex clusters, but there are many gaps, and some of which do not appear to be accidental.

This scenario makes it possible to study the processing of various types of consonant clusters. In the following, the focus is, first, on the distinction between existent (EX) and non-existent (NEX) clusters, and, second, on the distinction between well-formed (WF) and ill-formed (IF) clusters.

Wellformedness is defined for the present study by the notion of sonority: sounds are assumed to be inherently more or less sonorous, and a well-formed syllable presumedly consists of a sequence of sounds for which sonority tapers off from the vocalic nucleus towards both edges. For example, an English or German syllable spelt blank has two minimally sonorous sounds at the left and right edge $(/ \mathrm{b}, \mathrm{k} /)$, outside of two sounds with intermediate sonority values $(/ \mathrm{l}, \mathrm{y} /)$, and the most sonorous vowel /æ/ or /a/, respectively, in the nucleus or peak of the syllable. However, the sonority model of the syllable (see summary in Parker, 2012) has always been under debate; various definitions of the sonority scale have been proposed, and numerous exceptions to the sonority generalisation exist. The present paper attempts, inter alia, to study the role of sonority-related patterns in the processing of language.

For this purpose, the sonority scale given in (1) is assumed. It ranks classes of segments according to the degree of opening in the vocal tract required for the articulation of sounds of the respective class. " $<$ " is an abbreviation for "less sonorous than". We also give an example from each class.

(1) Sonority hierarchy

plosive $<$ affricate $<$ fricative $<$ nasal $<$ liquid $<$ glide $<$ vowel

\section{/b/ /ts/ /f/ /n/ /l/ $/ \mathrm{j} / \quad$ /i/}

This scale distinguishes five degrees of sonority for consonants and thus allows for a fine-grained distinction within consonant clusters. For example, a final cluster /fts/ with a fricative segment followed by the affricate /ts/ would be one adhering to the sonority hierarchy specified in (1), while a cluster/tsf/ would exemplify a violating cluster. While sonority as defined here is only one of the principles discussed for syllable phonology and is complemented by others such as identity avoidance (OCP), preference for CV syllables over all other types such as VC or CCV, it has certainly been considered both a central principle as well as a debated one.

Crucially, both types of clusters exist in languages such as German, and the existence of clusters violating the sonority principle may either be seen as evidence against this principle or as counterexamples to an otherwise valid principle (Parker, 2012). In any case, the fact that "illegal" clusters exist will be used in the present study.

The preparation of the stimuli used in the current experiment was guided by the consideration of cross-linguistic comparability of consonant subsets occurring in different languages. The experimental dataset was initially designed to allow for a comparison between German and Polish. Therefore, a list of possible consonant clusters was automatically generated with consonants common in the two languages. In the actual production of German, several consonants are syllabic in final CC-cluster position, for instance in $/ \mathrm{nl} /, / \mathrm{sn} /, / \mathrm{tr} /$ 
Table 1. Groups of clusters used.

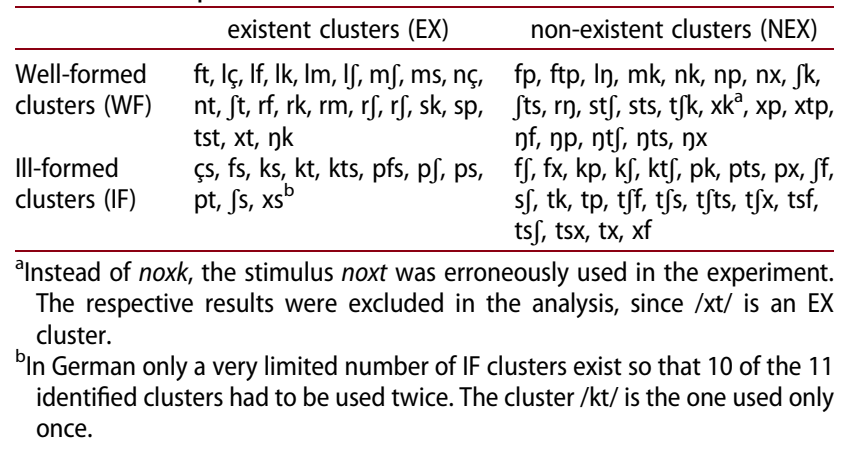

and were therefore excluded. The clusters were then classified into four groups adhering to our experimental criteria of sonority and existence. In other words, we created artificial word-stimuli with final consonant clusters either adhering to the sonority hierarchy as stated in (1) or not, and either existing in German or not. Since both types of clusters, that is, clusters violating the sonority hierarchy and clusters without violation, have both exemplars present in German and exemplars missing in German, we arrived at the sets of clusters presented in Table 1. The four groups derive from the crossing of two factors, namely formedness (well-formed vs. ill-formed) and existence (existent vs. non-existent).

One criterion for the selection of NEX clusters was their phonetic similarity with EX clusters. In other words NEX and EX clusters were matched in terms of voicing, place and manner of articulation as closely as possible. This requirement narrowed down the possible number of clusters. While it was not possible to control for the role of other potential features of clusters, stimuli included a wide range of possible clusters in all different groups. The identification of NEX, IF clusters was particularly difficult. In order to avoid to many repetitions of the same cluster in the creation of stimuli, we limited the number of clusters to be used to 21 per group so that we only had to duplicate 10 of the 11 clusters in the NEX, IF group.

\section{Experiment}

The experiment was based on 252 nonce words in a $2 \times 2$ design of formedness and existence resulting in 126 words with WF and 126 words with IF clusters as well as 126 words with EX and 126 words with NEX clusters (details on the construction of these nonce words are given below). The conditions were crossed so that our participants were presented with an equal number of items in four conditions represented in Table 2.

All stimuli were presented eight times in the course of the experiment, twice during a first EEG-session
Table 2. Conditions and number of stimuli.

\begin{tabular}{lll}
\hline & EX & NEX \\
\hline WF & 63 & 63 \\
IF & 63 & 63 \\
\hline
\end{tabular}

(pre-learning, EEG-1), four times during an online training and twice again during the subsequent second EEGsession (post-learning, EEG-2). The nonce words were introduced as names for unusual physical objects (see below).

The design of our experiment and stimuli allows for a number of predictions with respect to the individual factors of formedness and existence of the consonant clusters both, within each session (EEG-1 and EEG-2) and between sessions (in comparison of the two EEGsessions). Differences between individual and crossed conditions, and between the two sessions of the behavioural and neural responses are expected to become apparent in both processing and learning.

\section{Hypotheses}

For the behavioural data we expect the following results:

Overall, participants should be able to recollect wordpicture-pairs containing words with WF clusters more successfully than those with IF clusters, since simple structures may be easier to memorise than more complex structures. Additionally, recollection of words with EX clusters should be better than recollection of those with NEX clusters, because familiar items may be memorised more easily than non-familiar items. WFNEX-words and IF-EX-words should be identified correctly more often than IF-NEX-words however, less often than WF-EX-words since both, sonority and existence, are expected to influence recollection.

In the comparison of the two sessions, we expect recollection success to increase more significantly in items containing WF clusters compared to those with IF clusters, since words with IF clusters contain structures that cannot be derived from universally available primitives, and hence are harder to learn and to memorise. Previous work has shown that structures involving such violations affect language acquisition success in children and are more easily affected by language change (e.g. Kegl, Senghas, \& Coppola, 1999; Kirby, Cornish, \& Smith, 2008; Nevins, 2010; Smith \& Tsimpli, 1995). Secondly, we expect improvement to be significant in the correct identification of both, item pairs containing EX and NEX clusters. Due to extensive exposure during the study, NEX clusters should become less novel in the course of the experiment. Even brief auditory exposure has been shown to affect learnability of phonotactically legal and illegal words and nonce words (Bernard, 2011; Chambers et al., 2010). By the time of the second session, NEX clusters may be 
Table 3. Correctness rates for recollection (intra-experimental results; session 1).

\begin{tabular}{llll}
\hline WF & $>$ & & IF \\
EX & $>$ & NEX \\
WF-EX & WF-NEX/IF-EX & $>$ & IF-NEX \\
\hline
\end{tabular}

entrenched enough into the phonological representation of the subjects that non-existence will be far less important than wellformedness. These expectations are summarised in Tables 3 and 4.

For the EEG-results, we expect to find differences between conditions in the neural deflection of the electrophysiological response. However, since it is unclear which of the two major factors, sonority or existence, plays a more significant role in the processing of nonce words, we hypothesise neither about their relative contribution nor the size of possible observable effects in the electrophysiological response, but about differences in the components only. Nonce words, regardless of their adherence to phonotactic constraints, have been found to be associated with the N400 (Bentin et al., 1999; Domahs et al., 2009; Friedrich \& Friederici, 2005; Holcomb, 1988, 1993; Holcomb \& Neville, 1990; Kutas \& Hillyard, 1980; Kutas, Neville, \& Holcomb, 1987; Rossi et al., 2011). In the processing of licit and illicit nonce words, however, differences have been found in the electrophysiological signal in the form of a later positivity (LPC) (Domahs et al., 2009). Hence, words with IF clusters should evoke a late positive effect compared to those with WF clusters, whereas words with NEX clusters should elicit an early negativity effect compared to words with EX clusters. The latter, however, is expected to decrease in EEG-2 compared to EEG-1 due to extensive exposure.

These hypotheses will be tested by looking at the differences between EEG-reactions to the nonce words, and comparing the results obtained for the pre-learning and post-learning phase.

\section{Overview experiment}

Participants were exposed to nonce words as stimuli, and were asked to learn these words as names for simple objects. During the experiment, we recorded correct responses for word-picture-pairs and electrophysiological reactions (ERPs) to the auditory presentation of these stimuli in two successive experimental sessions set apart by an intermediate individual online training containing additional presentations of the stimuli.

Table 4. Correctness rates for recollection learning (interexperimental comparison; session 1 vs. session 2).

\begin{tabular}{llllll}
\hline WF & $>$ & & IF & & \\
EX & $=$ & & NEX & & \\
WF-EX $>$ & WF-NEX & $>$ & IF-EX $>$ & IF-NEX \\
\hline
\end{tabular}

\section{Participants}

Thirty right-handed native speakers of German (15 male, 15 female) with normal hearing and no reported visual problems participated in the experiment. One participant had to be excluded from the analysis of the EEGsessions for technical reasons so that the results presented below are based on the data of 29 participants. Subjects' mean age was 24 (ranging from 21 to 30 years), they were mostly registered students at the University of Marburg and paid for their participation.

\section{Material}

Two hundred and fifty-two stimuli were used in the experiment designed according to the criteria described above, monosyllabic nonce words with a CVCC structure. The critical section, that is, the final consonant cluster, was preceded by a CV string consisting of three different consonants and three different vowels. They were chosen as unmarked and sufficiently distinct from each other in German. Additionally, consonants differed in places (velar, alveolar, labial-dental) and manners (plosive, fricative, and nasal) of articulation, namely $g, f, n$. Vowels were distinct in height, frontness and roundness $(a, e, o)$. The resulting nine different CV sequences ( $g e, g a, g o, f e, f a$ fo and na, na, no) allowed for the presentation of each final cluster in three different contexts, as for example in /gektg/, /faktf/, and /noktg/ or /gasp/, /fosp/, and /nesp/.

The participants were presented with the total of 74 clusters, 21 clusters in three of the four conditions (WFEX; WF-NEX, IF-NEX) and 11 in the fourth condition (IF$E X)$. In German, a limited number of IF clusters exists so that only 11 IF-EX clusters were identified and used in the experiment. In order to include a comparable number of stimuli for each condition, 10 of the IF-EX clusters were doubled. Additionally, we increased the number of items for each condition by embedding them into three different CV contexts as detailed above, resulting in 63 stimuli per condition. Each stimulus word was spoken at a normal speech rate by a phonetically trained female native speaker of German from the Berlin area. Recordings took place in a soundproof cabin at the University of Marburg. The words were spoken in isolation and directly recorded onto a Mac computer, digitised at $44.1 \mathrm{kHz}$ with a 16-bit sampling rate (mono format). Since some of the clusters were articulatorily very demanding, recordings took place under the supervision of a phonetician who controlled for the clusters' authentic but clear pronunciation and to avoid unnaturally careful pronunciation. The individual stimuli were cut at the beginning and the end of the word (Amadeus Pro Version 2.1 (1523)) by a trained phonetician. 
The number of critical items used was 21 (types) $\times 2$ (EX vs. NEX) $\times 2$ (WF vs. IF) $\times 3$ (3 CV contexts), resulting in 252 items. The experimental stimuli were auditorily presented via loudspeakers as names for 252 objects, including an equal number of artefacts, rare animals, insects, reptiles, and plants respectively, drawn from various websites and presented subsequently to the respective auditory stimulus. Pictures of rare and unfamiliar items were chosen to ensure that it was unlikely that participants had any name for them. Familiarity was assessed in the authors' team. To maintain a highly comparable visual appearance, picture size was standardised $(425 \times 425$ pixels, $15 \times 15 \mathrm{~cm})$. The size of objects, plants, animals, and insects was held comparable in the pictures, and they were presented on a black screen.

\section{Experimental procedure}

We recorded behavioural and EEG-data in a sound proof cabin at the neurolinguistic laboratory of the Institute of German Linguistics at the University of Marburg. The experiment involved three parts, consisting of two EEGsessions with intermittent one or two days during which participants had to complete an online training on the internet-based learning and teaching platform Integriertes Lern-, Informations- und Arbeitskooperations-System (ILIAS) of the University of Marburg. Table 5 illustrates the course of the experiment.

Prior to the first EEG-session, subjects had to complete a training sequence in order to familiarise themselves with the task and the situation. The training sequence consisted of 21 practice trials, the equivalent to one block of the actual experiment. Instruction regarding the procedure was given prior to the training sequence and was followed by feedback, when necessary. The training sequence was repeated when subjects felt the need; however feedback on correct or incorrect responses in individual trials was not provided. The 2 EEG-sessions were identical for each individual subject and consisted of 12 blocks. However, each subject was provided with a different version of the experiment. We used twelve different block orderings to exclude order effects and effects of fatigue. In addition we reversed correct and incorrect pairings of words and pictures in half of the blocks. Furthermore, we avoided a handedness bias by assigning correct responses in these 12 versions to the left joystick button. In 12 duplicated versions we assigned the correct response button

Table 5. Time-course of the experiment.

\begin{tabular}{lcc}
\hline Day 1 & Day 2 (or 3) & Day 3 (or 4) \\
\hline Pre-learning EEG-1 & Web-based online training & Post-learning EEG-2 \\
\hline
\end{tabular}

to the right joystick button. This procedure leads to 24 different versions of the experiment.

The experiment took approximately 60 minutes including training sequence, breaks and a resting phase. During the experiment participants were comfortably seated in front of a computer screen. They were presented with the same set of 252 word-picture-pairs, divided into 12 short blocks. Between the blocks participants were allowed to take a short, self-paced break and a longer break took place after the 6th block. Each block consisted of two phases, a stimulus-presentation-phase and a response-elicitation-phase. During the presentation-phase, 21 word-picture-pairs were presented. The task here was to simply memorise as many of the word-picture-pairs as possible. The same set of 21 word-picture-pairs was presented in the immediately following elicitation-phase. However, half of the wordpicture-pairs in the elicitation-phase was matched as in the presentation-phase and half of them were incorrectly matched. During this phase, the picture was followed by a question mark, indicating to the participants the response-interval. The subjects' task was to decide whether the mapping of the word and the picture corresponded to that previously introduced in the presentation-phase by pressing the joystick buttons. The task for the participant in each trial thus was to learn a new name for an unusual object, which constitutes an ecologically valid verbal task. This ensured that participants would not focus explicitly on the phonotactic properties of the stimuli. The response-interval had a time-out of $2000 \mathrm{~ms}$. Pressing the joystick buttons triggered the next trial after 1500 ms. Each phase, namely the presentation- and the elicitation-phase, was initiated by an auditory presentation of a synthesised sine wave at $340 \mathrm{~Hz}$ for $500 \mathrm{~ms}$. The time-line of each presentationand elicitation-phase trial is illustrated in Tables 6 and 7.

On the day following the first EEG, participants were required to complete the web-based online training.

Table 6. Time-line of the presentation-phase.

\begin{tabular}{lcc}
\hline $\begin{array}{l}\text { Stimulus (auditory presentation) } \\
\text { accompanied by a fixation star (visual } \\
\text { presentation) }\end{array}$ & $\begin{array}{r}\text { Picture (visual } \\
\text { presentation) }\end{array}$ & $\begin{array}{r}\text { Blank } \\
\text { screen }\end{array}$ \\
\hline $1500 \mathrm{~ms}$ & $1500 \mathrm{~ms}$ & $1500 \mathrm{~ms}$ \\
\hline
\end{tabular}

Table 7. Time-line of the elicitation-phase.

\begin{tabular}{lccc}
\hline $\begin{array}{l}\text { Stimulus (auditory } \\
\text { presentation) }\end{array}$ & & & \\
$\begin{array}{l}\text { accompanied by a } \\
\text { fixation star (visual }\end{array}$ & $\begin{array}{c}\text { Picture (visual } \\
\text { presentation) }\end{array}$ & $\begin{array}{c}\text { Question mark } \\
\text { (visual presentation) } \\
\text { response-interval }\end{array}$ & $\begin{array}{c}\text { Blank } \\
\text { screen }\end{array}$ \\
\hline $1500 \mathrm{~ms}$ & $1500 \mathrm{~ms}$ & $\max 2000 \mathrm{~ms}$ & 1500 \\
& & & $\mathrm{~ms}$ \\
\hline
\end{tabular}


Subjects had to complete six chapters of a learning module, each followed by a test. We devised four different sets of tests differing with respect to word-picturematch and their order. The same word-picture-pairs as in the EEG-experiments were presented in blocks of 42 pairs followed by a test-phase during which participants had to indicate again, if the word-picture-pair is correct or incorrect. For each participant correct and incorrect pairs were reversed compared to those presented during the EEG-experiments, which means that correct pairs presented during the EEG-experiments were incorrect in the online training and vice versa. Both learningand elicitation-phase had to be completed twice during the online training and answers were recorded. The second EEG-experiment took place on the day following the training day, that is, two days after the first experiment with one exception where the second EEGsession took place three days after the EEG-1. The procedure and the experiment version for each participant was the exact same as in the first EEG-session during the second EEG-session.

\section{Phonetic analysis of stimuli}

Since we used only neologisms it is crucial to exclude potential artefacts due to differences in the phonetic realisation. Therefore, we compared the phonetic characteristics of mean fundamental frequency, mean intensity, and mean duration of the items produced.

Table 8 shows that the stimuli of the four conditions used in our experiment differ with respect to the phonetic parameter of mean fundamental frequency and duration. Intensity does not differ significantly between any of the four conditions used in the stimulus material. Although the differences in pitch between most of the conditions are "large" (approximately one standard deviation), psycholinguistic studies have revealed that sound signals have to differ in at least three semitones in order to be reliably discriminable by humans (e.g. 't Hart, 1981; 't Hart, Collier, \& Cohen, 1990, p. 29; Nooteboom, 1997). Even under the assumption of a perceptual threshold of $1.5 \mathrm{ST}$, as suggested by Rietveld and Gussenhoven $(1985$, p. 304), the differences in mean fundamental frequency observed in our stimulus material are equal or smaller.

Table 8. Phonetic parameters (means, standard deviations) for items in four conditions.

\begin{tabular}{lcll}
\hline Nonce word type & F0 $(\mathrm{Hz})$ & \multicolumn{1}{c}{ Intensity $(\mathrm{dB})$} & \multicolumn{1}{c}{ Duration $(\mathrm{sec})$} \\
\hline WF-EX $(n=63)$ & $201.8076( \pm 12.8)$ & $51.0401( \pm 5.1)$ & $0.8169( \pm 0.1)$ \\
WF-NEX $(n=63)$ & $200.3581( \pm 15.5)$ & $50.6044( \pm 6.1) \mathrm{dB}$ & $0.9444( \pm 0.12)$ \\
IF-EX $(n=63)$ & $212.8162( \pm 14.6)$ & $50.4801( \pm 3.7) \mathrm{dB}$ & $0.9823( \pm 0.1)$ \\
IF-NEX $(n=63)$ & $223.1418( \pm 17.1)$ & $50.5895( \pm 4.4) \mathrm{dB}$ & $0.9245( \pm 0.1)$ \\
\hline
\end{tabular}

"Large" (again, approximately one standard deviation) differences in duration were also found between all conditions with the exception of WF-NEX and both IF-EX and IF-NEX. Words with WF-EX clusters are shorter than words of all other conditions whereas words with IF-EX clusters are longest. The differences in duration range between $58 \mathrm{~ms}$ between IF-EX and IF-NEX and $166 \mathrm{~ms}$ between WF-EX and IF-EX with values of $128 \mathrm{~ms}$ (WFEX and WF-NEX) and $110 \mathrm{~ms}$ (WF-EX and IF-NEX) inbetween.

Although we do not assume these differences to influence the behavioural reactions to the consonant clusters, since word length can only be fully evaluated at the end of a word, and the properties of the consonant clusters will be processed much earlier, in fact already with the beginning of the vowel in the nucleus of the syllable, we provide an analysis of the influence of the phonetic properties (F0, intensity and duration) in the appendix. The acoustic variation between stimuli is partly modelled by the item term in the random effect structure for the EEG statistics. The effects in the more comprehensive model are largely the same as the ones presented here, but the additional model complexity has a corresponding increase in model interpretation.

\section{EEG and analysis}

EEG was recorded by means of $27 \mathrm{Ag}-\mathrm{AgCl}$ electrodes (F7, F3, Fz, F4, F8, FC5, FC1, FCZ, FC2, FC6, T7, C3, CZ, C4, T8, CP5, CP1, Cpz, CP2, CP6, P7, P3, Pz, P4, P8, POz) according to the $10 \%$ extension of the International 10-20 system. AFz electrode served as ground electrode, and the reference electrode during the experiment was located at the left mastoid, re-referenced off-line to linked mastoids. Impedances of electrodes were kept below $5 \mathrm{k} \Omega$. Electrodes above and below the participants' left eye as well as electrodes placed at the lateral canthus of both eyes (electrooculogram, EOG) recorded the vertical and horizontal eye movements respectively to control for eye-movement artefacts. For the EEG- and EOG-measurements, we used a BrainAmp amplifier (Brain Products, Germany). Recordings were carried out continuously with a digitisation rate of 500 $\mathrm{Hz}$, and filtered off-line with a bandpass filter from 0.16 to $30 \mathrm{~Hz}$ and trials with artefacts automatically rejected.

For the analysis of the EEG-data we used mixedeffects models with participants and items as crossed random factors, and session, region of interest (ROI), formedness, and existence for factors $(115,020$ total observations after automatic artefact rejection). This analysis was chosen over the more traditional separate subject and items analyses as it is less dependent on normality and sphericity assumptions, can cope well with 
missing data, and allows for the combined analysis of categorical and continuous predictors (e.g. Baayen, Davidson, \& Bates, 2008; Barr, 2008; Jaeger, 2008; Quené \& van den Bergh, 2004).

For the analysis of the EEG-sessions, only data from the elicitation-phase (and not the presentation-phase) were used. The presentation-phase was used to determine relevant time-windows in the EEG-signal for further analysis, thus avoiding violations of independence via "douple dipping". In order to focus as closely as possible on the word-final clusters and to exclude temporal phonetic variability introduced by the iteminitial CV-sequence, EEG-responses are analysed from the intensity peak of the nucleus of each stimulus. The nuclei were identified automatically using a PRAAT script (De Jong \& Wempe, 2009) and manually checked. Mean and range latency of the peak occurrence in the nucleus are provided in Table 9.

To determine the time-windows for further analysis, the single-trial data from the presentation-phase was divided into successive $50 \mathrm{~ms}$ time-windows. Two linear mixed-effects models were calculated, one for formedness and one for existence. For this exploratory portion, only random-slope models restricted to the electrode $\mathrm{Cz}$ were used to maintain computational tractability. ${ }^{1}$ The fixed effects consisted of a single term for timewindow as well as its interaction with either formedness or existence. Successive windows where the interaction term achieved $|t|>3$ were used to determine the windows for further analysis, resulting in two larger windows: $450-550 \mathrm{~ms}$ and $700-1050 \mathrm{~ms}$ post nucleus.

Using the windows determined from the presentationphase, hypothesis testing was conducted with the elicitation-phase. Each time-window was analysed in a separate model. The fixed-effect structure in each included all interactions between session, $\mathrm{ROI}$, existence and formedness. In keeping in line with the recommendations of Barr, Levy, Scheepers, and Tily (2013) for maximal model structure supported by the hypotheses being tested, the random-effect structure included main effects for session, existence and formedness. Interactions were excluded to maintain computational tractability. ROI was similarly omitted from the random slope for the additional reason of not being a main effect of interest. Keeping in line with previous results and our own lack

Table 9. Means, maximum, minimum and standard deviation of word length (in ms) and peak occurrence in ms and percentage in relation to the word length.

\begin{tabular}{llll}
\hline & Words in ms & Nucleus in ms & Nucleus \% \\
\hline Mean & 908 & 226 & 25 \\
Minimum & 568 & 82 & 8 \\
Maximum & 1243 & 728 & 86 \\
Standard deviation & 125 & 98 & 10 \\
\hline
\end{tabular}

of hypotheses concerning topography, we aimed for parsimony by only using two ROIs (anterior: FC1, FC2, FCZ, FC5, FC6; posterior: CP1, CP2, Cpz, CP5, CP6).

\section{Results}

The present paper explored the influence of consonantclusters' existence and formedness in the processing of speech applying the methods of ERP's. The aim of the study was to test whether both universal and language-specific regularities and, on the other hand, input may play a role in the learnability of clusters in nonce words. Our results reveal a biphasic pattern for the tested comparisons. In the following, we present the behavioural results followed by possible explanations of negativity and positivity EEG effects observed.

\section{Behavioural data}

Accuracy results (\%) are presented in Table 10 across conditions and experimental sessions. We performed a repeated measurements Three-way analysis of variance with formedness (two levels: WF and IF), existence (two levels: EX and NEX) and session (two levels: EEG-1 and EEG-2) as within-subject factors. Mauchly's test indicated that the assumption of sphericity had been violated, therefore degrees of freedom were corrected using Greenhouse-Geisser estimates of sphericity. We found a significant main effect for session $\left(F_{(1: 28)}=195.40\right.$; $p<.001$ ): overall, word-picture-pairs were significantly more often correctly identified in EEG-2 compared to EEG-1. We also found significant main effects for formedness $\left(F_{(1: 28)}=8.9 ; p<.006\right)$ and existence $\left(F_{(1: 28)}=10.06\right.$; $p<.004)$ as well as significant interactions between formedness and existence $\left(F_{(1: 28)}=15.35 ; p<.001\right)$ and existence and session $\left(F_{(1: 28)}=4.87 ; p<.036\right)$ (Table 10).

Post hoc tests showed that formedness did not impact on the correct identification of words with NEX clusters $\left(F_{(1: 28)}=0.58 ; p<.452\right)$ but that there was a significant improvement in EEG-2 compared to EEG-1 $\left(F_{(1: 28)}=\right.$ $193.08 ; p<.001)$. Words with EX clusters however differed depending on their adherence to sonority in that words with WF-EX clusters were identified correctly more often

Table 10. Response accuracy (means, standard deviations) for items in four conditions and two experimental sessions.

\begin{tabular}{lllll}
\hline Formedness & Existence & EEG-session & Mean correct response \% & SD \\
\hline IF & EX & EEG-1 & 67.2 & 47 \\
IF & EX & EEG-2 & 84.1 & 37 \\
IF & NEX & EEG-1 & 67.1 & 47 \\
IF & NEX & EEG-2 & 85.7 & 35 \\
WF & EX & EEG-1 & 71.3 & 45 \\
WF & EX & EEG-2 & 88.7 & 32 \\
WF & NEX & EEG-1 & 65.7 & 47 \\
$W F$ & NEX & EEG-2 & 85.8 & 35 \\
\hline
\end{tabular}


than those with IF-EX $\left(F_{(1: 28)}=26.28 ; p<.001\right)$. There was a significant improvement in EEG-2 compared to EEG-1 $\left(F_{(1: 28)}=148.42 ; p<.001\right)$ as expected due to the observed main effect. In other words, learning took place regardless of the conditions, confirming previous findings on phonotactic learning of attested and unattested sequences and constraints (Bernard, 2011; Karlinsky, 2012; Koo \& Cole, 2006). All of these studies show that learning takes place even after very limited exposure. It appears worthwhile to ask the question why there are still stable patters to observe across languages and over times or even across an individual's language in her lifetime. The main effect of formedness therefore suggests that, in line with Berent, Steriade, Lennertz, and Vaknin (2007) and Redford (2008), universal constraints modulate phonotactic learning. Existence of consonant combinations attested in German on the other hand did not aid the recollection of words with IF clusters. Reaction times are not meaningful since subjects were advised to respond only during the time when the question mark appeared on screen following the presentation of the picture.

\section{ERP data}

The results presented in this section are based on 115,020 observations from 63 items and 29 subjects. Figure 1 illustrates the results obtained from the analysis of the EEG recordings in the first time-window (450-550 ms after nucleus onset) by means of an image displaying deviations from mean voltage on a grey-scale on the basis of the linear mixed-effect model specified above. Figure 1 is divided into four larger squares and each of those into additional four smaller squares. Descriptions on $x$-axes and $y$-axes specify the conditions defining

Table 11. Main effects and interactions (both bold) in the timewindow 450-550 ms.

\begin{tabular}{llll} 
& & \multicolumn{1}{c}{$\begin{array}{c}\text { Std. } \\
\text { error }\end{array}$} & $\begin{array}{c}t^{-} \\
\text {value }\end{array}$ \\
\hline (Intercept) & -2.8970 & 0.4687 & -6.18 \\
Session 2 & $\mathbf{1 . 9 1 8 2}$ & $\mathbf{0 . 3 3 6 8}$ & $\mathbf{5 . 7 0}$ \\
ROI (posterior) & -0.2223 & 0.1170 & -1.90 \\
existence (NEX) & -0.2045 & 0.2285 & -0.90 \\
Formedness (IF) & $-\mathbf{0 . 9 5 0 2}$ & $\mathbf{0 . 1 8 2 9}$ & $-\mathbf{5 . 2 0}$ \\
Session 2: ROI (posterior) & -0.2695 & 0.1645 & -1.64 \\
Session 2: existence (NEX) & -0.0463 & 0.1561 & -0.30 \\
ROI (posterior): existence (NEX) & $\mathbf{0 . 3 4 4 1}$ & $\mathbf{0 . 1 6 6 0}$ & $\mathbf{2 . 0 7}$ \\
Session 2: formedness (IF) & -0.2046 & 0.1554 & -1.32 \\
ROI (posterior): formedness (IF) & $\mathbf{0 . 4 6 9 0}$ & $\mathbf{0 . 1 6 5 2}$ & $\mathbf{2 . 8 4}$ \\
Existence (NEX): formedness (IF) & $\mathbf{0 . 4 7 0 4}$ & $\mathbf{0 . 1 5 6 1}$ & $\mathbf{3 . 0 1}$ \\
Session 2: ROI (posterior): existence (NEX) & -0.2992 & 0.2338 & -1.28 \\
Session 2: ROI (posterior): formedness (IF) & -0.0738 & 0.2328 & -0.32 \\
Session 2: existence (NEX): formedness (IF) & 0.1038 & 0.2204 & 0.47 \\
ROI (posterior): existence (NEX): formedness & -0.3500 & 0.2338 & -1.50 \\
(IF) & & & \\
Session 2: ROI (posterior): existence (NEX): & 0.1927 & 0.3302 & 0.58 \\
formedness (IF) & & & \\
\hline
\end{tabular}

each of these squares. Negativity is depicted by intensity of the grey-scale: the darker the more negative.

Overall, the two squares on the right-hand side, that is, measurements recorded during the second EEG are brighter than those on the left-hand side recorded during the first EEG. This illustrates a higher negativity for ERPs recorded during the first session, as reflected in the main effect for session ( $t=5.70$; positive because a negativity in the first session is more positive than no negativity in the second session). For ROI ( $t=-1.90)$ we only found a weak effect in that the negativity effect was more pronounced in the posterior region than in the anterior region. For formedness a main effect was found $(t=-5.20)$, with a negativity effect for IF clusters. Significant interactions were found between ROI and existence $(t=2.07), \mathrm{ROI}$ and formedness $(t=2.84)$ and existence and formedness $(t=3.01)$. The interaction between $\mathrm{ROI}$ and existence results from the observation that the negativity effect for NEX clusters compared to EX clusters was less pronounced in the posterior region compared to the anterior region. The interaction between ROI and formedness results from a larger negativity effect for IF clusters in the anterior regions compared to the posterior region.

The last and most crucial observation is an interaction between formedness and existence, as illustrated in the ERP plots presented in Figure 2. The negativity effect for words with IF clusters (red lines) is more pronounced in those that contain EX clusters (solid line), whereas in words with WF clusters (blue lines) the effect is reversed. Words with WF and NEX clusters (blue dotted line) elicit the stronger effect. The relevant time-window is highlighted as early negativity in the plots.

Coefficient estimates (change in ERP mean in microvolt between conditions) standard error and $t$-values are presented in Table 11. The conditions in parentheses represent the baseline. $T$-values of $> \pm 2$ indicate significant differences (Baayen et al., 2008).

Figure 3 illustrates the results obtained in the second time-window of 700-1050 ms from nucleus onset, in a way analogous to Figure 1.

The main effect for session was also found in the later time-window from 700 to $1050 \mathrm{~ms}(t=2.28)$. In addition, we found a main effect for ROI as well, but the effect was reversed compared to the earlier time-window, with a positivity effect for the posterior region $(t=8.39)$. Significant interactions were found for session and existence $(t=3.28)$, existence and formedness $(t=6.44)$ and for session, existence, and formedness $(t=-4.18)$. This latter three-way interaction results from the fact that the interaction between formedness and existence is more pronounced in the second session compared to the first. 


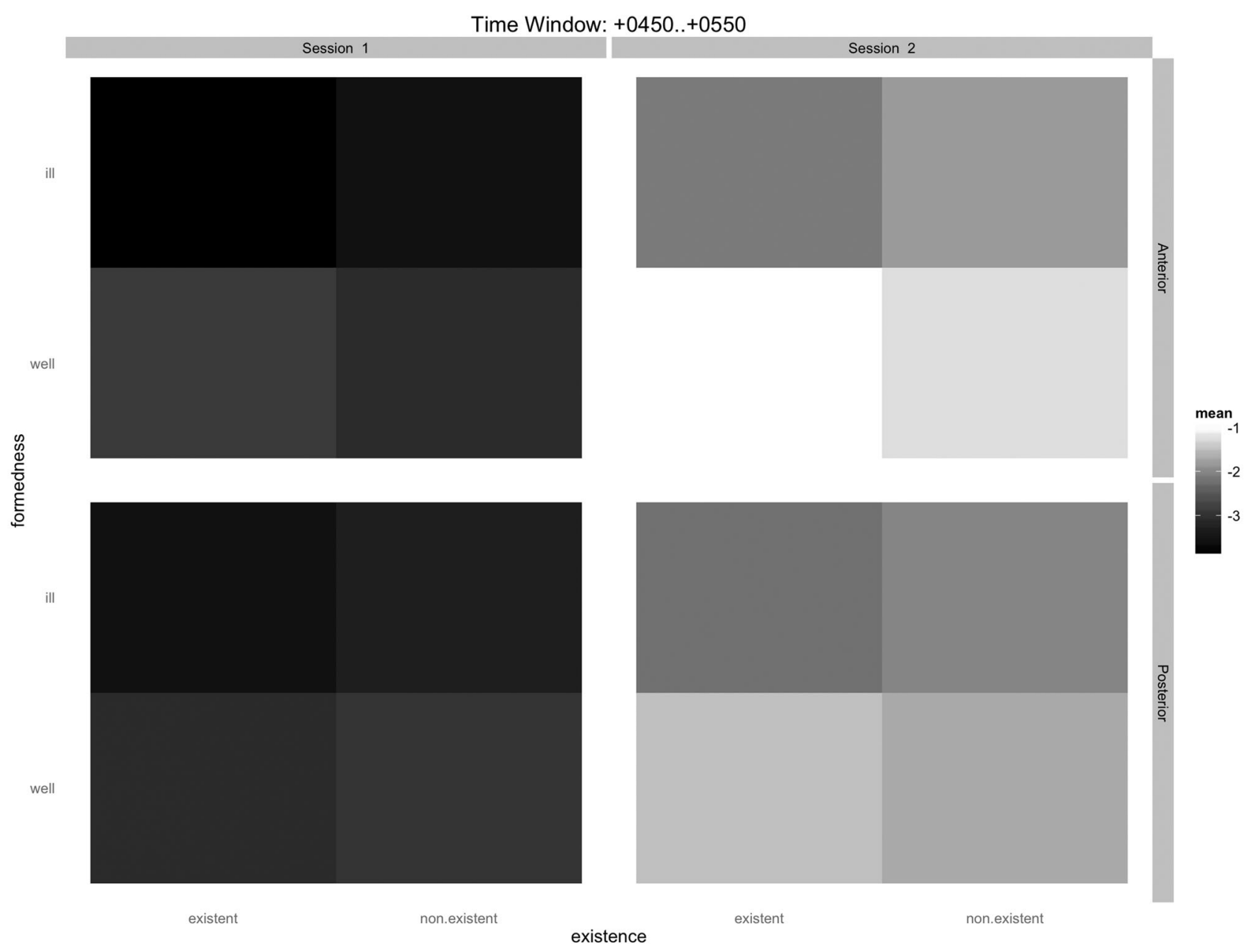

Figure 1. Mean micro volt differences for existence, formedness, ROI and session in the time-window $450-550$ ms. Negativity is depicted by intensity of the grey-scale.

The significant interaction between formedness and existence is also illustrated in the EEG plots given in Figure 2. The relevant time-window is highlighted as late positivity in the plots. IF clusters in general (red lines) are more positive than WF clusters, but the effect is more pronounced in IF-NEX clusters (dotted red line). Words with WF and NEX clusters (blue dotted line) elicit the weaker effect. Table 12 presents the statistical analysis for the
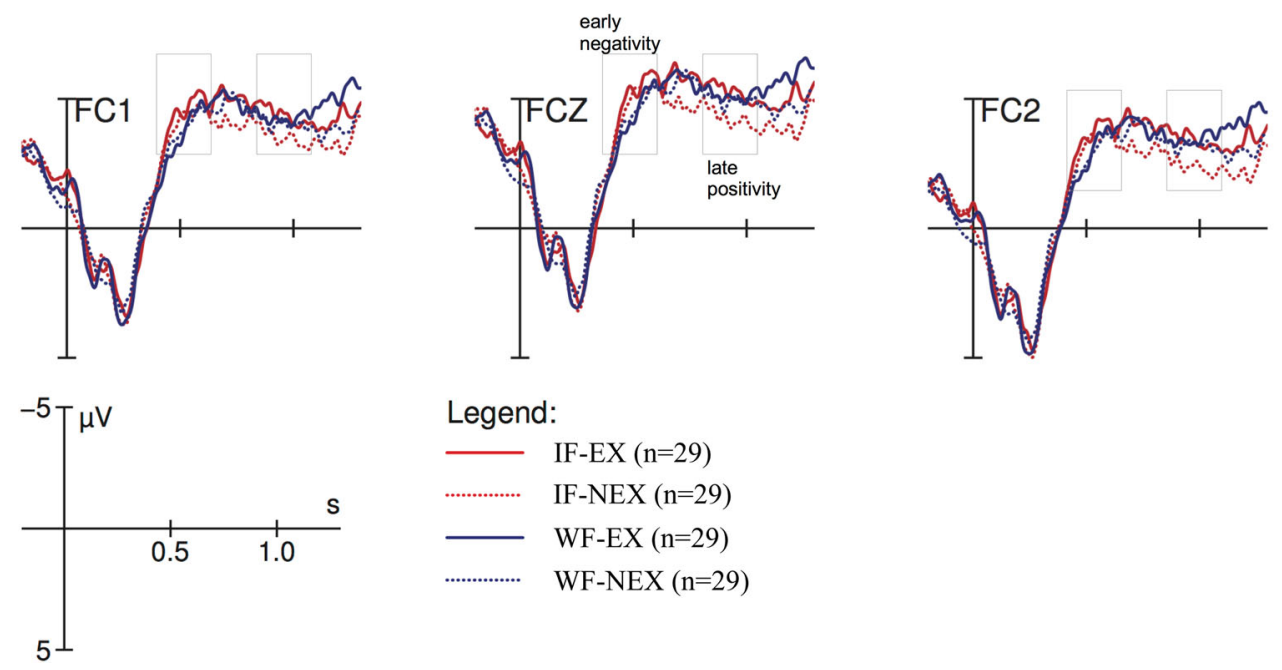

Legend:

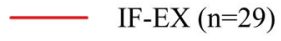

IF-NEX $(\mathrm{n}=29)$

WF-EX $(n=29)$

WF-NEX (n=29)

Figure 2. Grand average ERPs (three frontal electrodes) for experimental conditions existence, formedness. 
Table 12. Main effects and interactions (both bold) in the timewindow 700-1050 ms.

\begin{tabular}{|c|c|c|c|}
\hline & Estimate & $\begin{array}{l}\text { Std. } \\
\text { error }\end{array}$ & $t$-value \\
\hline (Intercept) & -4.13947 & 0.38723 & -10.69 \\
\hline Session 2 & 0.77532 & 0.34027 & 2.28 \\
\hline ROI (posterior) & 0.81029 & 0.09656 & 8.39 \\
\hline Existence (NEX) & -0.00722 & 0.17143 & -0.04 \\
\hline Formedness (IF) & -0.28219 & 0.18239 & -1.55 \\
\hline Session 2: ROI (posterior) & -0.05637 & 0.13574 & -0.42 \\
\hline Session 2: existence (NEX) & 0.42304 & 0.12884 & 3.28 \\
\hline ROI (posterior): existence (NEX) & 0.19589 & 0.13695 & 1.43 \\
\hline Session 2: formedness (IF) & 0.17704 & 0.12827 & 1.38 \\
\hline ROI (posterior): formedness (IF) & 0.14031 & 0.13629 & 1.03 \\
\hline Existence (NEX): formedness (IF) & 0.83009 & 0.12880 & 6.44 \\
\hline Session 2: ROI (posterior): existence (NEX) & -0.17463 & 0.19293 & -0.91 \\
\hline Session 2: ROI (posterior): formedness (IF) & -0.00159 & 0.19211 & 0.01 \\
\hline $\begin{array}{l}\text { Session 2: existence (NEX): formedness } \\
\text { (IF) }\end{array}$ & -0.76038 & 0.18184 & -4.18 \\
\hline $\begin{array}{l}\text { ROI (posterior): existence (NEX): } \\
\text { formedness (IF) }\end{array}$ & -0.10002 & 0.19292 & -0.52 \\
\hline $\begin{array}{l}\text { Session 2: ROI (posterior): existence (NEX): } \\
\text { formedness (IF) }\end{array}$ & 0.19414 & 0.27244 & 0.71 \\
\hline
\end{tabular}

second time-window. As in the previous Table 10, baseline conditions are provided in parentheses.

\section{Discussion}

The higher correctness rates achieved in EEG-2 indicate that the experimental design was an appropriate one for the observation of learnability. Furthermore, the results demonstrate that wellformedness plays a more significant role for the entrenchment of new words into the lexicon than occurrence in the German language. It may be that individuals can draw on frequency effects when they are exposed to linguistic structures that adhere to universal constraints of phonotactics, but not to those that violate them.

The less pronounced differences in the second session compared to the first session, in both time-windows, indicate that learning took place and reflects an increased familiarity with the non-words.

Considering the first time-window (450-550 ms), we interpret the negativity effect as an instance of the N400 effect. The main effect for formedness results from an increased negativity for words with IF clusters compared to those with WF clusters. Previous research (Domahs et al., 2009) has shown that nonce words can

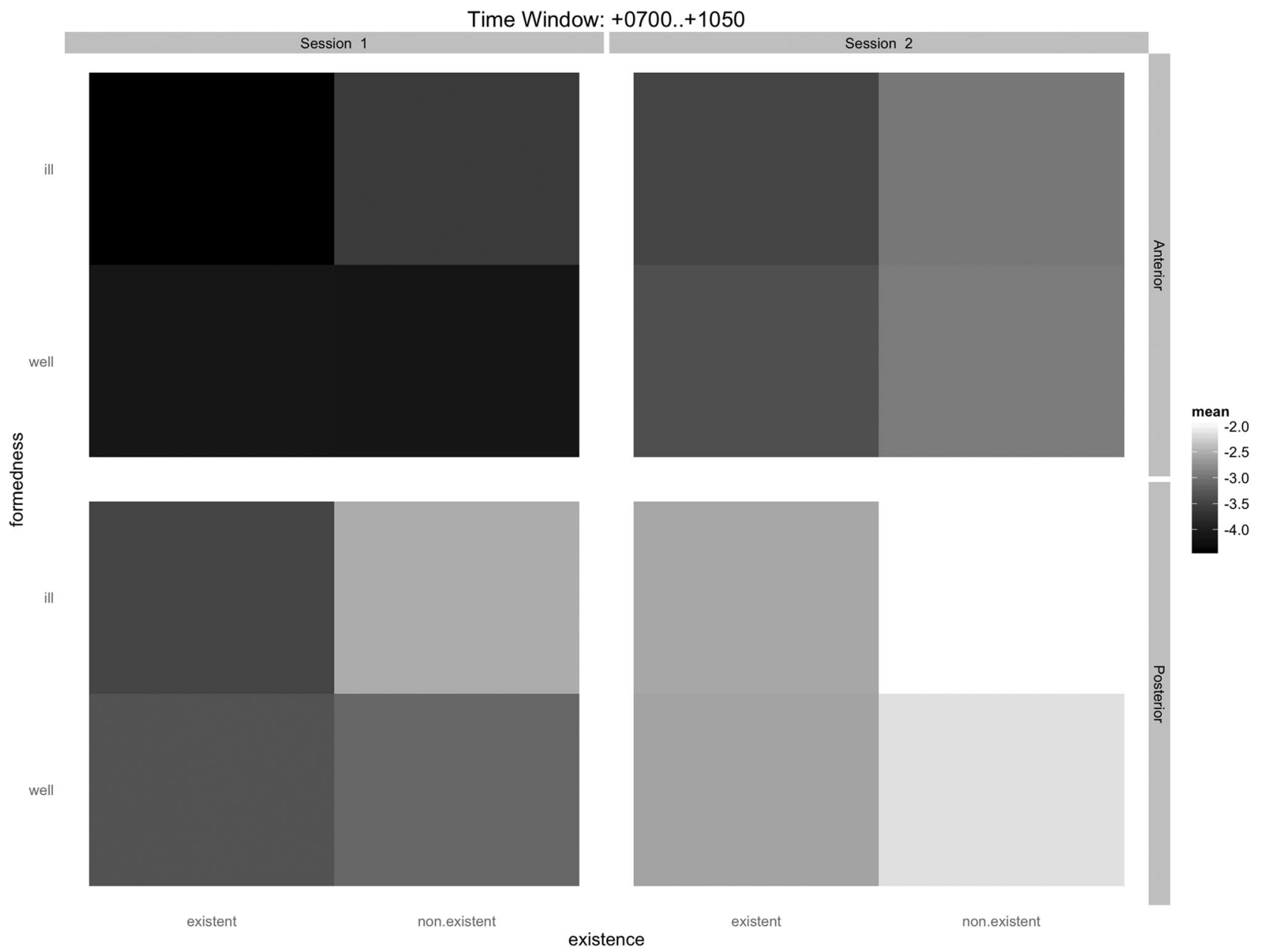

Figure 3. Mean micro volt differences for existence, formedness, ROI and session in the time-window 700-1050 ms. Negativity is depicted by intensity of the grey-scale. 
elicit a N400, even before an item is recognised and lexical meaning can be retrieved. Moreover, the N400 effect has been shown to be elicited automatically or in secondary tasks (see Kutas \& Federmeier, 2011, for review). The negativity effect observed in the current study thus reflects pre-lexical phonological analysis of the clusters (Deacon, Dynowska, Ritter, \& Grose-Fifer, 2004). Our results with respect to sonority as a principle of wellformedness can be interpreted as confirming such form-based processing, in line with the findings for the role of sonority in phonological development (e.g. Yavas \& Gogate, 1999), and for aphasic speech (e.g. Stenneken, Bastiaanse, Huber, \& Jacobs, 2005).

Crucially, it appears that, contrary to our hypotheses, the early processing of words with EX clusters does not differ from the processing of words with NEX clusters (although this effect was significant in the models with acoustic parameters, s. Appendix). This may be due to the fact that all of our stimuli were nonce words. In Domahs et al. (2009), nonce words as opposed to real words elicited a negativity effect, regardless of their adherence to or violation of phonotactic constraints. It has been found previously that only those items that trigger lexicalsemantic integration mechanisms, that is, the integration of words into a sentence (Friederici, Pfeifer, \& Hahne, 1993), or that of a picture into a story, elicit an N400 in the ERP (West \& Holcomb, 2002). The lack of such a negativity effect in our data may be due to the fact that only nonce words were used in the current experiment. Nonce words in general are known to elicit a larger N400 component (Kutas \& Federmeier, 2011), so that we suspect that the small phonological contrast is diminished compared to the larger lexical effect. Therefore, it is not surprising that we do not find an $\mathrm{N} 400$ for the distinction between words with EX and NEX clusters.

However, it is not the case that (non-) existence does not play a role at all, but that it has rather a differential effect in interaction with $\mathrm{ROI}$ and formedness. The interaction between formedness and existence can be explained by means of unfulfilled expectations. IF clusters, very rare in German, would rather be expected to be NEX, whereas WF clusters would rather be expected to be EX. Conflicting information of two competing factors influencing the processing of words may increase processing costs. In other words, IF clusters are more marked than WF clusters, reflected in the main effect for formedness. The processing of marked structures may be more difficult when they exist and lead to deeper processing (Bentin et al., 1999; Domahs et al., 2009).

The observation that the early negativity is localised more fronto-centrally is in accordance with previous findings (e.g. Friederici et al., 2006) and leads to the prediction of a subsequent centro-parietal positivity, confirmed in the main effect observed for ROI in the second time-window (700-1050 ms). Even though, this result is in line with previous findings locating a late positivity at the centro-parietal electrode sites in recollectionstudies (Finnigan, Humphreys, Dennis, \& Geffen, 2002), the relation between pronounced ERP-responses and specific brain areas is rather speculative due to the poor spatial resolution of ERPs. Therefore, any firm and reliable conclusion would have to be drawn from results obtained with methods offering higher spatial resolution.

In the second time-window, we also found a main effect for session. The positivity of the ERP-responses was more pronounced in the second session compared to the first session. Studies involving a study-testdesign and tasks of incidental as well as intentional encoding frequently reported a late positivity component (LPC) (for review see Friedman \& Johnson, 2000). Our paradigm involves intentional encoding of word-picture-pairs so that the increased positivity from session 1 to session 2 indicates that more items are recognised during the second session. Furthermore, the LPC was more pronounced in the processing of words with NEX clusters compared to those with EX clusters in the second session. These results are in line with previous findings where the posterior late positivity has been attributed to recognition or familiarity (Rugg et al., 1998; Rugg \& Curran, 2007). We also observed a significant interaction between formedness and existence in the second time-window. Examining the ERP plots reveals that whether or not a cluster is well-formed does not seem to play as an important role for existent clusters, but does for non-existent clusters. These findings support previous results showing the modulation of the LPC depending on the evaluation of phonological violation, for example, violations of rhythmic structures (Bohn, Knaus, Wiese, \& Domahs, 2013) and phonotactic constraints (Bentin et al., 1999; Domahs et al., 2009). However, Bohn et al. (2013, p. 769) showed in an investigation of rhythmic irregularities in the processing of natural speech that the late positivity is not only tasksensitive. In a comparison of stimuli involving rhythmical and lexical violations with stimuli involving a rhythmic violation only, the authors showed that the size of the positivity effect is related to the relative ease of evaluation, that is, the easier the evaluation of an item as unnatural or deviant from expectancy, the stronger the positivity effect. Our results, with a strongest LPC effect for words with IF-NEX clusters, confirm this conclusion. In addition, our findings show that this effect does not only occur in explicit evaluation tasks but also in secondary tasks involving primarily the recollection of testitems. 


\section{Conclusions}

The aim of the study was to examine the learnability and the processing of the phonological constraint of sonority as well as the influence of actual existence of consonant clusters by means of electrophysiological measurements. The present results show that both, formedness and existence, play a significant role in the processing of word-like items in German. The data confirm that violations of the phonotactic constraint of sonority are processed differently from non-violating items. Our study obtained results from a word-picture-matching task, as well as from ERP data, thus complementing results obtained by Berent et al. (2014) from an fMRI study.

In addition, our results show that the existence of clusters affects their processing as well, but in a more indirect way. In accordance with previous findings our results show that the implicit knowledge of phonological universals as well as frequent exposure influences the online-processing and facilitates the learning of structures that comply with such principles.

We therefore have found evidence for both views argued for in current phonological theory (and often seen as mutually exclusive): phonological knowledge is based both on abstract principles such as sonority and on input patterns such as prior existence.

\section{Note}

1. The anti-conservative nature of these models is irrelevant as any false positives will be caught by the more focused hypothesis testing and will indeed thus work as more conservative by increasing the span of data to be tested. The electrode $\mathrm{Cz}$ was taken as being representative as all of the hypothesized components have an at least partially central distribution.

\section{Acknowledgements}

We are grateful to Ina Bornkessel-Schlesewsky, Ulrike Domahs, Ekaterina Starikova and other colleagues at the Department of German Linguistics, Marburg University, for helpful discussions.

\section{Disclosure statement}

No potential conflict of interest was reported by the authors.

\section{Funding}

This work was supported by the LOEWE initiative of the state of Hesse, Germany (Fundierung linguistischer Basiskategorien).

\section{References}

Baayen, R. H., Davidson, D. J., \& Bates, D. M. (2008). Mixed-effects modeling with crossed random effects for subjects and items. Journal of Memory and Language, 59(4), 390-412. doi:10.1016/j.jml.2007.12.005

Barr, D. J. (2008). Analyzing 'visual world' eyetracking data using multilevel logistic regression. Journal of Memory and Language, 59, 457-474. doi:10.1016/j.jml.2007.09.002

Barr, D. J., Levy, R., Scheepers, C., \& Tily, H. J. (2013). Randomeffects structure for confirmatory hypothesis testing: Keep it maximal. Journal of Memory and Language, 68, 255-278. doi:10.1016/j.jml.2012.11.001

Bentin, S., Mouchetant-Rostaing, Y., Giard, M. H., Echallier, J. F., \& Pernier, J. (1999). ERP manifestations of processing printed words at different psycholinguistic levels: Time course and scalp distribution. Journal of Cognitive Neuroscience, 11(3), 235-260. doi:10.1162/089892999563373

Berent, I., Pan, H., Zhao, X., Epstein, J., Bennett, M. L., Deshpandes, V., \& Stern, E. (2014). Language universals engage Broca's area. Plos One, 9(4), e95155. doi:10.1371/ journal.pone.0095155

Berent, I., Steriade, D., Lennertz, T., \& Vaknin, V. (2007). What we know about what we have never heard: Evidence from perceptual illusions. Cognition, 104(3), 591-630. doi:10.1016/j. cognition.2006.05.015

Bernard, A. (2011). An onset is an onset: Evidence from abstraction of newly-learned phonotactic constraints (master thesis). University of Illinois, Urbana-Champaign. Retrieved from http://hdl.handle.net/2142/29780

Bohn, K., Knaus, J., Wiese, R., \& Domahs, U. (2013). The influence of rhythmic (ir)regularities on speech processing: Evidence from an ERP study on German phrases. Neuropsychologia, 51, 760-771. doi:10.1016/j.neuropsychologia.2013.01.006

Chambers, K. E., Onishi, K. H., \& Fisher, C. (2010). A vowel is a vowel: Generalizing newly learned phonotactic constraints to new contexts. Journal of Experimental Psychology: Learning, Memory, and Cognition, 36(3), 821-828. doi.org/ $10.1037 / \mathrm{a} 0018991$

Chomsky, N., \& Halle, M. (1968). The sound pattern of English. New York: Harper \& Row.

Clements, G. N. (1990). The role of the sonority cycle in core syllabification. In J. Kingston \& M. E. Beckman (Eds.), Papers in laboratory phonology I: Between the grammar and the physics of speech (pp. 283-333). Cambridge: Cambridge University Press.

De Jong, N., \& Wempe, T. (2009). PRAAT script to detect syllable nuclei and measure speech rate automatically. Behavior Research Methods, 41, 385-390. doi:10.3758/BRM.41.2.385

Deacon, D., Dynowska, A., Ritter, W., \& Grose-Fifer, J. (2004). Repetition and semantic priming of nonwords: Implications for theories of N400 and word recognition. Psychophysiology, 41(1), 60-74. doi: 10.1111/1469-8986.00120

Dell, G. S., Reed, K. D., Adams, D. R., \& Meyer, A. S. (2000). Speech errors, phonotactic constraints, and implicit learning: A study of the role of experience in language production. Journal of Experimental Psychology. Learning, Memory, and Cognition, 26 (6), 1355-1367. doi.org/10.1037/0278-7393.26.6.1355

Domahs, U., Kehrein, W., Knaus, J., Wiese, R., \& Schlesewsky, M. (2009). Event-related potentials reflecting the processing of phonological constraint violations. Language and Speech, 52(4), 415-435. doi:10.1177/0023830909336581 
Dziubalska-Kołaczyk, K. (2002). Phonology without the syllable: A study in the natural framework. Poznań: Motivex.

Ellis, N. C., \& Larsen-Freeman, D. (2006). Language emergence: Implications for applied linguistics - introduction to the special issue. Applied Linguistics, 27(4), 558-589. doi:10. 1093/applin/aml028

Endress, A. D., \& Hauser, M. D. (2010). Word segmentation with universal prosodic cues. Cognitive Psychology, 61, 177-199. doi:10.1016/j.cogpsych.2010.05.001

Endress, A. D., \& Mehler, J. (2010). Perceptual constraints in phonotactic learning. Journal of Experimental Psychology: Human Perception and Performance, 36(1), 235-250. doi.org/10.1037/ a0017164

Finnigan, S., Humphreys, M. S., Dennis, S., \& Geffen, G. (2002). ERP 'old/new' effects: Memory strength and decisional factor(s). Neuropsychologia, 40, 2288-2304. doi:10.1016/ S0028-3932(02)00113-6

Fowler, C. A. (1993). The structure of English syllables and polysyllables. Journal of Memory and Language, 32(1), 115-140. doi:10.1006/jmla.1993.1007

Friederici, A. D. (1995). The time-course of syntactic activation during language processing: A model-based on neuropsychological and neurophysiological data. Brain and Language, 50(3), 259-281. doi:10.1006/brln.1995.1048

Friederici, A. D. (2002). Towards a neural basis of auditory sentence processing. Trends in Cognitive Sciences, 6(2), 78-84. doi:10.1016/S1364-6613(00)01839-8

Friederici, A. D., Bahlmann, J., Heim, S., Schubotz, R. I., \& Anwander, A. (2006). The brain differentiates human and non-human grammars: Functional localization and structural connectivity. Proceedings of the National Academy of Sciences of the United States of America, 103(7), 2458-2463. doi:10. 1073/pnas.0509389103

Friederici, A. D., Pfeifer, E., \& Hahne, A. (1993). Event-related brain potentials during natural speech processing: Effects of semantic, morphological and syntactic violations. Cognitive Brain Research, 1, 183-192. doi:10.1016/0926-6410 (93)90026-2

Friedman, D., \& Johnson, R. E. (2000). Event-related potential (ERP) studies of memory encoding and retrieval: A selective review. Microscopy Research and Technique, 51, 6-28.

Friedrich, M., \& Friederici, A. D. (2005). Phonotactic knowledge and lexical-semantic processing in one-year-olds: Brain response to words and nonsense words in picture contexts. Journal of Cognitive Neuroscience, 17(11), 1785-1802. doi:10. 1162/089892905774589172

Frisch, S., \& Schlesewsky, M. (2005). The resolution of case conflicts from a neurophysiological perspective. Cognitive Brain Research, 25, 484-498. doi:10.1016/j.cogbrainres.2005. 07.010

Goad, H., \& Rose, Y. (2004). Input elaboration, head faithfulness and evidence for representation in the acquisition of left-edge clusters in West Germanic. In R. Kager, J. Pater, \& W. Zonneveld (Eds.), Fixing priorities: Constraints in phonological acquisition (pp. 109-157). Cambridge: CUP.

Goldrick, M., \& Larson, M. (2008). Phonotactic probability influences speech production. Cognition, 107(3), 1155-1164. doi:10.1016/j.cognition.2007.11.009

Hagoort, P. (2003). Interplay between syntax and semantics during sentence comprehension: ERP effects of combining syntactic and semantic violations. Journal of Cognitive Neuroscience, 15 (6), 883-99. doi:10.1162/089892903322370807
Hall, A. T. (1992). Syllable structure and syllable related processes in German. Tübingen: Niemeyer Verlag.

't Hart, J. (1981). Differential sensitivity to pitch distance, particularly in speech. Journal of the Acoustical Society of America, 69, 811-821. doi: 10.1121/1.385592

't Hart, J., Collier, C., \& Cohen, A. (1990). A perceptual study of intonation: An experimental-phonetic approach to speech melody. Cambridge: Cambridge University Press.

Henke, E., Kaisse, E. M., \& Wright, R. (2012). Is the sonority principle an epiphenomenon? In S. Parker (Ed.), The sonority controversy (pp. 65-100). Berlin: Walter de Gruyter.

Holcomb, P. (1988). Automatic and attentional processing: An event-related brain potential analysis of semantic priming. Brain and Language, 35, 66-85. doi:10.1016/0093-934X(88) 90101-0

Holcomb, P. J. (1993). Semantic priming and stimulus degradation: Implications for the role of the $\mathrm{N} 400$ in language processing. Psychophysiology, 30, 47-61. doi: 10.1111/j.14698986.1993.tb03204.x

Holcomb, P., \& Neville, H. (1990). Auditory and visual semantic priming in lexical decision: A comparison using eventrelated brain potentials. Language and Cognitive Processes, 5, 281-312. doi:10.1080/01690969008407065

Jaeger, T. F. (2008). Categorical data analysis: Away from ANOVAs (transformation or not) and towards logit mixed models. Journal of Memory and Language, 59, 434-446. doi. org/10.1016/j.jml.2007.11.007

Jusczyk, P. W., Luce, P. A., \& Charles-Luce, J. (1994). Infants' sensitivity to phonotactic patterns in the native language. Journal of Memory and Language, 33, 630-645. doi:10.1006/ jmla.1994.1030

Kabak, B., \& Idsardi, W. J. (2007). Perceptual distortions in the adaptation of English consonant clusters: Syllable structure or consonantal contact constraints? Language and Speech, 50, 23-52. doi:10.1177/00238309070500010201

Kager, R., Pater, J., \& Zonneveld, W. (2004). Constraints in phonological acquisition. Cambridge: Cambridge University Press.

Karlinsky, N. (2012). Learning phonotactic constraints in the production of non-native consonant clusters, as reflected in speech errors. Urbana-Champaign: University of Illinois. Retrieved from http://hdl.handle.net/2142/42251

Kegl, J., Senghas, A., \& Coppola, M. (1999). Creation through contact: Sign language emergence and sign language change in Nicaragua. In M. DeGraff (Ed.), Language creation and language change: Creolization, diachrony, and development (pp. 179-237). Cambridge, MA: MIT Press.

Kirby, S., Cornish, H., \& Smith, K. (2008). Cumulative cultural evolution in the laboratory: An experimental approach to the origins of structure in human language. Proceedings of the National Academy of Sciences, 105, 10681-10686. doi: 10.1073/pnas.0707835105

Kirk, C., \& Demuth, K. (2005). Asymmetries in the acquisition of word-initial and word-final consonant clusters. Journal of Child Language, 32, 709-734. doi:10.1017/S0305000905007130

Koo, H., \& Cole, J. (2006). On learnability and naturalness as constraints on phonological grammar. In A. Botinis (Ed.), Proceedings of ISCA tutorial and research workshop on experimental linguistics (pp. 174-177). Athens, Greece: University of Athens.

Kuhl, P. K. (2010). Brain mechanisms in early language acquisition. Neuron, 67, 713-727. doi:10.1016/j.neuron.2010.08.038 
Kutas, M., \& Federmeier, K. D. (2011). Thirty years and counting: Finding meaning in the N400 component of the event-related brain potential (ERP). The Annual Review of Psychology, 62, 621-647. doi:10.1146/annurev.psych.093008.131123

Kutas, M., \& Hillyard, S. A. (1980). Reading senseless sentences: Brain potentials reflect semantic incongruity. Science, 207, 203-204.

Kutas, M., Neville, H. J., \& Holcomb, P. J. (1987). A preliminary comparison of the $\mathrm{N} 400$ response to semantic anomalies during reading, listening and signing. In R. J. Ellingson, N. M. F. Murray, \& A. M. Halliday (Eds.), The London symposia, electroencephalography and clinical neurophysiology (pp. 325-330). Amsterdam: Elsevier.

Levelt, C., Schiller, N., \& Levelt, W. (2000). The acquisition of syllable types. Language Acquisition, 8, 237-64. doi:10.1207/ S15327817LA0803_2

Levitt, A., Healy, A. F., \& Fendrich, D. W. (1991). Syllable-internal structure and the sonority hierarchy: Differential evidence from lexical decision, naming, and reading. Journal of Psycholinguistic Research, 20, 337-363.

MacWhinney, B. (1999). Emergent language. Functionalism and Formalism in Linguistics, 1, 361-386.

Mattys, S. L., \& Jusczyk, P. W. (2001). Phonotactic cues for segmentation of fluent speech by infants. Cognition, 78, 91121. doi:10.1016/S0010-0277(00)00109-8

Mattys, S. L., Jusczyk, P. W., Luce, P. A., \& Morgan, J. L. (1999). Phonotactic and prosodic effects on word segmentation in infants. Cognitive Psychology, 38(4), 465-494. doi:10.1006/ cogp.1999.0721

McCarthy, J. J. (1986). OCP effects: Gemination and antigemination. Linguistic Inquiry, 17, 207-263.

Meerendonk, N., Chwilla, D. J., \& Kolk, H. H. J. (2013). States of indecision in the brain: ERP reflections of syntactic agreement violations versus visual degradation. Neuropsychologia, 51(8), 1383-1396. doi:10.1016/j.neuropsychologia.2013.03.025

Moreton, E. (2002). Structural constraints in the perception of English stop-sonorant clusters. Cognition, 84, 55-71. doi:10. 1016/S0010-0277(02)00014-8

Munson, B. (2001). Phonological pattern frequency and speech production in adults and children. Journal of Speech, Language, and Hearing Research, 44, 778-92. doi:10.1044/ 1092-4388

Nespor, M., Peña, M., \& Mehler, J. (2003). On the different roles of vowels and consonants in speech processing and language acquisition. Lingue e linguaggio, 2.2, 203-230. doi:10.1418/10879

Nevins, A. (2010). Two case studies in phonological universals: A view from artificial grammars. Biolinguistics, 4, 218-233.

Nobre, A. C., \& McCarthy, G. (1994). Language related ERPs: Scalp distributions and modulation by word-type and semantic priming. Journal of Cognitive Neuroscience, 6, 233255. doi:10.1162/jocn.1994.6.3.233

Nooteboom, S. G. (1997). The prosody of speech: Melody and rhythm. In W. J. Hardcastle \& J. Laver (Eds.), The handbook of phonetic sciences (pp. 640-673). Oxford: Blackwell.

Norris, D., McQueen, J. M., Cutler, A., \& Butterfield, S. (1997). Segmentation of continuous speech using phonotactics. Journal of Memory and Language, 39, 21-46. doi:10.1006/ jmla.1998.2568

Odden, D. (1986). On the role of the obligatory contour principle in phonological theory. Language, 62, 353-383.
Parker, S. (Ed.). (2012). The sonority controversy. Berlin: Walter de Gruyter.

Quené, H., \& van den Bergh, H. (2004). On multi-level modeling of data from repeated measures designs: A tutorial. Speech Communication, 43, 103-121. doi:10.1016/j.specom.2004.02.004

Redford, M. A. (2008). Production constraints on learning novel onset phonotactics. Cognition, 107, 785-816. doi:10.1016/j. cognition.2007.11.014

Rietveld, A. C. M., \& Gussenhoven, C. (1985). On the relation between pitch excursion size and prominence. Journal of Phonetics, 13, 299-308.

Rossi, S., Jürgenson, I. B., Hanulíková, A., Telkmeyer, S., Wartenburger, I., \& Obrig, H. (2011). Implicit processing of phonotactic cues: Evidence from electrophysiological and vascular responses. Journal of Cognitive Neuroscience, 23(7), 1752-1764. doi:10.1162/jocn.2010.21547

Rugg, M. D., \& Curran, T. (2007). Event-related potentials and recognition memory. Trends in Cognitive Sciences, 11, 251257. doi:10.1016/j.tics.2007.04.004

Rugg, M. D., Mark, R. E., Walla, P., Schloerscheidt, A. M., Birch, C. S., \& Allan, K. (1998). Dissociation of the neural correlates of implicit and explicit memory. Nature, 392, 595-598. doi:10. 1038/33396

Seidl, A., Cristià, A., Bernard, A., \& Onishi, K. H. (2009). Allophonic and phonemic contrasts in infants' learning of sound patterns. Language Learning and Development, 5(3), 191-202. doi:10.1080/15475440902754326

Shatzman, K. B., \& McQueen, J. M. (2006a). Prosodic knowledge affects the recognition of newly acquired words. Psychological Science, 17(5), 327-372. doi:10.1111/j.14679280.2006.01714.x

Shatzman, K. B., \& McQueen, J. M. (2006b). Segment duration as a cue to word boundaries in spoken-word recognition. Perception and Psychophysics, 68(1), 1-16.

Sievers, E. (1876/1901). Grundzüge der Lautphysiologie zur Einführung in das Studium der Lautlehre der indogermanischen Sprache (4th ed.). Leipzig: Breitkopf \& Härtel.

Smith, N. V. (1973). The acquisition of phonology: A case study. London: University Press.

Smith, N. V., \& Tsimpli, I. M. (1995). The mind of a savant: Language learning and modularity. Oxford: Blackwell.

Stenneken, P., Bastiaanse, R., Huber, W., \& Jacobs, A. M. (2005). Syllable structure and sonority in language inventory and aphasic neologisms. Brain and Language, 95, 280-292. doi:10.1016/j.bandl.2005.01.013

Steriade, D. (1999). Alternatives to syllable-based accounts of consonantal phonotactics. In O. Fujimura, B. Joseph, \& B. Palek (Eds.), Proceedings of the 1998 linguistics and phonetics conference (pp. 205-242). Prag: Karolinum Press.

Treiman, R. (1984). On the status of final consonant clusters in English syllables. Journal of Verbal Learning and Verbal Behavior, 23, 343-356. doi:10.1016/S0022-5371(84)90237-8

Treiman, R., Fowler, C. A., Gross, J., Berch, D., \& Weatherston, S. (1995). Syllable structure or word structure? Evidence for onset and rime units with disyllabic and trisyllabic stimuli. Journal of Memory and Language, 34(1), 132-155. doi:10. 1006/jmla.1995.1007

Warker, J. A., \& Dell, G. S. (2006). Speech errors reflect newly learned phonotactic constraints. Journal of Experimental Psychology: Learning, Memory, and Cognition, 32(2), 387398. doi:10.1037/0278-7393.32.2.387 
West, W. C., \& Holcomb, P. J. (2002). Event-related potentials during discourse-level semantic integration of complex pictures. Cognitive Brain Research, 13, 363-375. doi:10.1016/ S0926-6410(01)00129-X

Whitney, W. D. (1865). On the relation of vowels and consonants. Journal of the American Oriental Society, 8, 357-373.

Wiese, R. (1988). Silbische und lexikalische Phonologie. Tübingen: Niemeyer Verlag.

Wiese, R. (2000). The phonology of German. Oxford: Oxford University Press.

Yavas, M., \& Gogate, L. J. (1999). Phonological awareness in children: A function of sonority. Journal of Psycholinguistic Research, 28, 245-260.

\section{Appendix}

Here, we present expanded models including acoustic parameters. These models generally provided a significantly better fit than the models presented in the main text (as indicated by AIC, BIC and likelihood-ratio tests); however, due to the large number of parameters, they are much more difficult to understand. As such, we presented the simpler models in the main text. The general structure of the effects between the basic and full-acoustic models is largely unchanged. Indeed, some effects increased in strength: in the second time-window we observed a main effect for existence and the interactions between session with existence and existence with formedness increased. None of the acoustic parameters showed a main effect, although interactions are present, which are generally more difficult to interpret and generally subject to higher levels of Type I error.

The main effect for session did fall below the significance threshold, but this is neither particularly problematic nor surprising. It is not problematic because the same stimuli were used for both sessions and as such, there is no confound between session and the acoustic parameters. It is not surprising because the effect was barely over the significance threshold even in the simpler model $(t=2.28)$, and the addition of further parameters to a model tends to reduce the power for the individual effects (the same amount of data has to spread its "explanatory ability" over more estimates). Crucially, the first-order interactions between session and the experimental manipulation remain.

Interestingly, existence became significant in both time-windows. This result is line with the hypotheses presented above. N400 amplitude is known to vary inversely with lexical frequency and nonce words are per definition minimally frequent. In terms of the LPC, while not predicted, the effect is still compatible with our hypotheses.

Table A1. Main effects and interactions (bold) in the time-window 450-550 ms including phonetic properties (F0, intensity and duration).

\begin{tabular}{|c|c|c|c|}
\hline & Estimate std. & Error & $t$-value \\
\hline (Intercept) & $-3.20 e+02$ & $1.68 e+02$ & -1.90 \\
\hline Session 2 & $2.06 e+02$ & $1.76 e+02$ & 1.17 \\
\hline ROI (posterior) & $1.63 e+02$ & $1.78 e+02$ & 0.92 \\
\hline Existence (NEX) & $5.74 e+02$ & $2.57 e+02$ & 2.23 \\
\hline Formedness (IF) & $1.68 e+03$ & $4.28 e+02$ & 3.94 \\
\hline F0 & $4.08 e+00$ & $3.29 e+00$ & 1.24 \\
\hline Duration & $3.87 e+02$ & $2.13 e+02$ & 1.82 \\
\hline Intensity & $1.61 e+00$ & $8.39 e-01$ & 1.92 \\
\hline Session 2: ROI (posterior) & $-2.35 e+02$ & $2.47 e+02$ & -0.95 \\
\hline Session 2: existence (NEX) & $-8.60 e+02$ & $2.66 e+02$ & -3.24 \\
\hline ROI (posterior): existence (NEX) & $-3.87 e+02$ & $2.64 \mathrm{e}+02$ & -1.47 \\
\hline Session 2: formedness (IF) & $-5.38 e+02$ & $4.27 e+02$ & -1.26 \\
\hline ROI (posterior): formedness (IF) & $-4.06 e+02$ & $4.25 e+02$ & -0.95 \\
\hline Existence (NEX): formedness (IF) & $-2.06 e+03$ & $5.78 e+02$ & -3.57 \\
\hline Session 2: F0 & $-3.91 e+00$ & $3.45 e+00$ & -1.13 \\
\hline ROI (posterior): F0 & $-3.13 e+00$ & $3.47 e+00$ & -0.90 \\
\hline Existence (NEX): F0 & $-9.34 e+00$ & $5.05 e+00$ & -1.85 \\
\hline Formedness (IF): F0 & $-3.07 e+01$ & $8.60 e+00$ & -3.57 \\
\hline Session 2: duration & $-2.10 \mathrm{e}+02$ & $2.22 e+02$ & -0.94 \\
\hline ROI (posterior): duration & $-1.84 e+02$ & $2.23 e+02$ & -0.83 \\
\hline Existence (NEX): duration & $-6.54 e+02$ & $3.03 e+02$ & -2.16 \\
\hline Formedness (IF): duration & $-1.87 e+03$ & $4.47 e+02$ & -4.18 \\
\hline F0: duration & $-4.80 \mathrm{e}+00$ & $4.18 e+00$ & -1.15 \\
\hline Session 2: intensity & $-1.07 e+00$ & $8.76 e-01$ & -1.22 \\
\hline ROI (posterior): intensity & $-8.43 e-01$ & $8.82 e-01$ & -0.96 \\
\hline Existence (NEX): intensity & $-2.77 e+00$ & $1.27 e+00$ & -2.18 \\
\hline Formedness (IF): intensity & $-8.16 e+00$ & $2.01 e+00$ & -4.06 \\
\hline Fo: intensity & $-2.11 e-02$ & $1.64 \mathrm{e}-02$ & -1.28 \\
\hline Duration: intensity & $-2.00 e+00$ & $1.05 e+00$ & -1.89 \\
\hline Session 2: ROI (posterior): existence (NEX) & $4.45 e+02$ & $3.71 e+02$ & 1.20 \\
\hline
\end{tabular}


Table A1. Continued.

\begin{tabular}{|c|c|c|c|}
\hline & Estimate std. & Error & $t$-value \\
\hline Session 2: ROI (posterior): formedness (IF) & $7.28 e+02$ & $5.98 e+02$ & 1.22 \\
\hline Session (2): existence (NEX): formedness (IF) & $6.93 e+02$ & $5.69 e+02$ & 1.22 \\
\hline ROI (posterior): existence (NEX): formedness (IF) & $1.42 e+02$ & $5.60 e+02$ & 0.25 \\
\hline Session 2: ROI (posterior): F0 & $4.62 e+00$ & $4.83 e+00$ & 0.96 \\
\hline Session (2): existence (NEX): F0 & $1.65 e+01$ & $5.25 e+00$ & 3.14 \\
\hline ROI (posterior): existence (NEX): F0 & $7.58 e+00$ & $5.22 \mathrm{e}+00$ & 1.45 \\
\hline Session 2: formedness (IF): F0 & $8.96 e+00$ & $8.61 e+00$ & 1.04 \\
\hline ROI (posterior): formedness (IF): F0 & $7.82 e+00$ & $8.57 e+00$ & 0.91 \\
\hline Existence (NEX): formedness (IF): F0 & $3.89 e+01$ & $1.15 e+01$ & 3.40 \\
\hline Session 2: ROI (posterior): duration & $2.77 e+02$ & $3.11 e+02$ & 0.89 \\
\hline Session 2: existence (NEX): duration & $9.54 e+02$ & $3.14 e+02$ & 3.04 \\
\hline Session 2: existence (NEX): duration & $4.13 e+02$ & $3.12 e+02$ & 1.32 \\
\hline ROI (posterior): existence (NEX): duration & $4.88 e+02$ & $4.51 e+02$ & 1.08 \\
\hline Session 2: formedness (IF): duration & $4.55 e+02$ & $4.51 e+02$ & 1.01 \\
\hline ROI (posterior): formedness (IF): duration & $2.28 e+03$ & $6.03 e+02$ & 3.78 \\
\hline Existence (NEX): formedness (IF): duration & $3.93 e+00$ & $4.36 e+00$ & 0.90 \\
\hline Session 2: F0: duration & $3.41 e+00$ & $4.39 e+00$ & 0.78 \\
\hline ROI (posterior): F0: duration & $1.04 \mathrm{e}+01$ & $5.96 e+00$ & 1.74 \\
\hline Existence (NEX): F0: duration & $3.39 e+01$ & $8.99 e+00$ & 3.78 \\
\hline Formedness (IF): F0: duration & $1.20 \mathrm{e}+00$ & $1.23 e+00$ & 0.98 \\
\hline Session 2: ROI (posterior): intensity & $4.01 e+00$ & $1.31 e+00$ & 3.07 \\
\hline Session 2: existence (NEX): intensity & $1.89 e+00$ & $1.30 \mathrm{e}+00$ & 1.46 \\
\hline ROI (posterior): existence (NEX): intensity & $2.90 e+00$ & $2.01 e+00$ & 1.44 \\
\hline Session 2: formedness (IF): intensity & $1.94 \mathrm{e}+00$ & $2.01 e+00$ & 0.97 \\
\hline ROI (posterior): formedness (IF): intensity & $9.67 e+00$ & $2.69 e+00$ & 3.60 \\
\hline Existence (NEX): formedness (IF): intensity & $2.06 e-02$ & $1.71 e-02$ & 1.20 \\
\hline Session 2: F0: intensity & $1.64 e-02$ & $1.72 e-02$ & 0.95 \\
\hline ROI (posterior): F0: intensity & $4.45 e-02$ & $2.49 e-02$ & 1.79 \\
\hline Existence (NEX): F0: intensity & $1.49 e-01$ & $4.05 e-02$ & 3.69 \\
\hline Formedness (IF): F0: intensity & $1.10 \mathrm{e}+00$ & $1.10 \mathrm{e}+00$ & 1.00 \\
\hline Session 2: duration: intensity & $9.54 \mathrm{e}-01$ & $1.10 \mathrm{e}+00$ & 0.86 \\
\hline ROI (posterior): duration: intensity & $3.19 e+00$ & $1.49 e+00$ & 2.13 \\
\hline Existence (NEX): duration: intensity & $9.07 e+00$ & $2.11 e+00$ & 4.30 \\
\hline Formedness (IF): duration: intensity & $2.54 e-02$ & $2.07 e-02$ & 1.23 \\
\hline F0: duration: intensity & $-2.80 e+02$ & $7.88 e+02$ & -0.36 \\
\hline Session 2: ROI (posterior): existence (NEX): formedness (IF) & $-8.36 e+00$ & $7.33 e+00$ & -1.14 \\
\hline Session 2: ROI (posterior): existence (NEX): F0 & $-1.38 e+01$ & $1.20 \mathrm{e}+01$ & -1.15 \\
\hline Session 2: ROI (posterior): formedness (IF): F0 & $-1.11 e+01$ & $1.13 e+01$ & -0.98 \\
\hline Session 2: existence (NEX): formedness (IF): F0 & $-2.64 e+00$ & $1.11 e+01$ & -0.24 \\
\hline ROI (posterior): existence (NEX): formedness (IF): F0 & $-4.89 e+02$ & $4.38 e+02$ & -1.12 \\
\hline Session 2: ROI (posterior): existence (NEX): duration & $-7.96 e+02$ & $6.33 e+02$ & -1.26 \\
\hline Session 2: ROI (posterior): formedness (IF): duration & $-7.00 e+02$ & $6.02 e+02$ & -1.16 \\
\hline Session 2: existence (NEX): formedness (IF): duration & $-1.48 e+02$ & $5.96 e+02$ & -0.25 \\
\hline ROI (posterior): existence (NEX): formedness (IF): duration & $-5.42 e+00$ & $6.11 e+00$ & -0.89 \\
\hline Session 2: ROI (posterior): F0: duration & $-1.81 e+01$ & $6.20 e+00$ & -2.93 \\
\hline Session 2: existence (NEX): F0: duration & $-7.99 e+00$ & $6.17 e+00$ & -1.29 \\
\hline ROI (posterior): existence (NEX): FO: duration & $-7.92 e+00$ & $9.10 \mathrm{e}+00$ & -0.87 \\
\hline Session 2: formedness (IF): F0: duration & $-8.71 e+00$ & $9.09 e+00$ & -0.96 \\
\hline ROI (posterior): formedness (IF): F0: duration & $-4.28 e+01$ & $1.20 e+01$ & -3.58 \\
\hline Existence (NEX): formedness (IF): F0: duration & $-2.19 e+00$ & $1.82 \mathrm{e}+00$ & -1.20 \\
\hline Session 2: ROI (posterior): existence (NEX): intensity & $-3.50 \mathrm{e}+00$ & $2.82 e+00$ & -1.24 \\
\hline Session 2: ROI (posterior): formedness (IF): intensity & $-3.66 e+00$ & $2.64 e+00$ & -1.38 \\
\hline Session 2: existence (NEX): formedness (IF): intensity & $-8.55 e-01$ & $2.61 e+00$ & -0.33 \\
\hline ROI (posterior): existence (NEX): formedness (IF): intensity & $-2.37 e-02$ & $2.40 \mathrm{e}-02$ & -0.99 \\
\hline Session 2: ROI (posterior): F0: intensity & $-7.65 e-02$ & $2.58 e-02$ & -2.96 \\
\hline Session 2: existence (NEX): F0: intensity & $-3.69 \mathrm{e}-02$ & $2.56 \mathrm{e}-02$ & -1.44 \\
\hline ROI (posterior): existence (NEX): FO: intensity & $-4.95 e-02$ & $4.06 e-02$ & -1.22 \\
\hline Session 2: formedness (IF): F0: intensity & $-3.75 e-02$ & $4.05 e-02$ & -0.93 \\
\hline Existence (NEX): formedness (IF): F0: intensity & $-1.82 e-01$ & $5.34 e-02$ & -3.42 \\
\hline Session 2: ROI (posterior): duration: intensity & $-1.41 e+00$ & $1.53 e+00$ & -0.92 \\
\hline Session 2: existence (NEX): duration: intensity & $-4.46 e+00$ & $1.54 e+00$ & -2.89 \\
\hline ROI (posterior): existence (NEX): duration: intensity & $-2.02 e+00$ & $1.53 e+00$ & -1.32 \\
\hline Session 2: formedness (IF): duration: intensity & $-2.64 e+00$ & $2.13 e+00$ & -1.24 \\
\hline ROI (posterior): formedness (IF): duration: intensity & $-2.19 e+00$ & $2.14 e+00$ & -1.02 \\
\hline Existence (NEX): formedness (IF): duration: intensity & $-1.07 e+01$ & $2.81 e+00$ & -3.81 \\
\hline Session 2: F0: duration: intensity & $-2.07 e-02$ & $2.16 e-02$ & -0.96 \\
\hline ROI (posterior): F0: duration: intensity & $-1.79 e-02$ & $2.17 e-02$ & -0.82 \\
\hline Existence (NEX): F0: duration: intensity & $-5.02 e-02$ & $2.94 \mathrm{e}-02$ & -1.71 \\
\hline Formedness (IF): F0: duration: intensity & $-1.65 e-01$ & $4.24 e-02$ & -3.89 \\
\hline Session 2: ROI (posterior): existence (NEX): formedness (IF): F0 & $4.74 \mathrm{e}+00$ & $1.57 e+01$ & 0.30 \\
\hline Session 2: ROI (posterior): existence (NEX): formedness (IF): F0 & $3.33 e+02$ & $8.39 e+02$ & 0.40 \\
\hline
\end{tabular}


Table A1. Continued.

\begin{tabular}{|c|c|c|c|}
\hline & Estimate std. & Error & $t$-value \\
\hline Session 2: ROI (posterior): existence (NEX): F0: duration & $9.10 e+00$ & $8.67 e+00$ & 1.05 \\
\hline Session 2: ROI (posterior): formedness (IF): F0: duration & $1.51 e+01$ & $1.28 \mathrm{e}+01$ & 1.19 \\
\hline Session 2: existence (NEX): formedness (IF): F0: duration & $1.09 e+01$ & $1.20 \mathrm{e}+01$ & 0.91 \\
\hline ROI (posterior): existence (NEX): formedness (IF): F0: duration & $2.75 e+00$ & $1.19 e+01$ & 0.23 \\
\hline Session 2: ROI (posterior): existence (NEX): formedness (IF): intensity & $1.64 \mathrm{e}+00$ & $3.67 e+00$ & 0.45 \\
\hline Session 2: ROI (posterior): existence (NEX): F0: intensity & $4.11 e-02$ & $3.60 e-02$ & 1.14 \\
\hline Session 2: ROI (posterior): formedness (IF): F0: intensity & $6.67 e-02$ & $5.69 e-02$ & 1.17 \\
\hline Session 2: existence (NEX): formedness (IF): F0: intensity & $6.01 e-02$ & $5.26 \mathrm{e}-02$ & 1.14 \\
\hline ROI (posterior): existence (NEX): formedness (IF): F0: intensity & $1.57 e-02$ & $5.20 e-02$ & 0.30 \\
\hline Session 2: ROI (posterior): existence (NEX): duration: intensity & $2.40 \mathrm{e}+00$ & $2.15 e+00$ & 1.12 \\
\hline Session 2: ROI (posterior): formedness (IF): duration: intensity & $3.82 e+00$ & $3.00 e+00$ & 1.28 \\
\hline Session 2: existence (NEX): formedness (IF): duration: intensity & $3.69 e+00$ & $2.81 e+00$ & 1.31 \\
\hline ROI (posterior): existence (NEX): formedness (IF): duration: intensity & $9.04 e-01$ & $2.78 e+00$ & 0.33 \\
\hline Session 2: ROI (posterior): F0: duration: intensity & $2.75 e-02$ & $3.02 \mathrm{e}-02$ & 0.91 \\
\hline Session 2: existence (NEX): F0: duration: intensity & $8.45 e-02$ & $3.04 e-02$ & 2.78 \\
\hline ROI (posterior): existence (NEX): F0: duration: intensity & $3.89 e-02$ & $3.03 e-02$ & 1.28 \\
\hline Session 2: formedness (IF): F0: duration: intensity & $4.43 e-02$ & $4.30 \mathrm{e}-02$ & 1.03 \\
\hline ROI (posterior): formedness (IF): F0: duration: intensity & $4.19 e-02$ & $4.31 e-02$ & 0.97 \\
\hline Existence (NEX): formedness (IF): F0: duration: intensity & $2.01 e-01$ & $5.58 e-02$ & 3.60 \\
\hline Session 2: ROI (posterior): existence (NEX): formedness (IF): F0: duration & $-5.70 e+00$ & $1.67 e+01$ & -0.34 \\
\hline Session 2: ROI (posterior): existence (NEX): formedness (IF): F0: intensity & $-2.83 e-02$ & $7.32 e-02$ & -0.39 \\
\hline Session 2: ROI (posterior): existence (NEX): formedness (IF): duration: intensity & $-1.89 e+00$ & $3.92 e+00$ & -0.48 \\
\hline Session 2: ROI (posterior): existence (NEX): F0: duration: intensity & $-4.46 e-02$ & $4.25 e-02$ & -1.05 \\
\hline Session 2: ROI (posterior): formedness (IF): F0: duration: intensity & $-7.29 e-02$ & $6.04 e-02$ & -1.21 \\
\hline Session 2: existence (NEX): formedness (IF): F0: duration: intensity & $-5.94 e-02$ & $5.58 e-02$ & -1.06 \\
\hline ROI (posterior): existence (NEX): formedness (IF): F0: duration: intensity & $-1.66 \mathrm{e}-02$ & $5.54 e-02$ & -0.30 \\
\hline Session 2: ROI (posterior): existence (NEX): formedness (IF): F0: duration: intensity & $3.29 e-02$ & $7.81 e-02$ & 0.42 \\
\hline
\end{tabular}

Table A2. Main efects and interactions (bold) in the time-window 700-1050 ms including phonetic properties (F0, intensity and duration).

\begin{tabular}{|c|c|c|c|}
\hline & Estimate & Std. error & $t$ \\
\hline (Intercept) & $-3.16 e+02$ & $1.37 e+02$ & -2.30 \\
\hline Session 2 & $2.01 e+01$ & $1.47 e+02$ & 0.14 \\
\hline ROI (posterior) & $3.53 e+02$ & $1.46 e+02$ & 2.41 \\
\hline Existence (NEX) & $5.71 e+02$ & $2.12 e+02$ & 2.69 \\
\hline Formedness (IF) & $1.61 e+03$ & $3.54 e+02$ & 4.56 \\
\hline F0 & $5.18 e+00$ & $2.69 e+00$ & 1.93 \\
\hline Duration & $3.10 e+02$ & $1.73 e+02$ & 1.79 \\
\hline Intensity & $1.57 e+00$ & $6.84 e-01$ & 2.30 \\
\hline Session 2: ROI (posterior) & $-3.81 e+02$ & $2.04 \mathrm{e}+02$ & -1.87 \\
\hline Session 2: existence (NEX) & $-1.90 e+02$ & $2.22 e+02$ & -0.86 \\
\hline ROI (posterior): existence (NEX) & $-3.71 e+02$ & $2.18 e+02$ & -1.70 \\
\hline Session 2: formedness (IF) & $-3.99 e+02$ & $3.57 e+02$ & -1.12 \\
\hline ROI (posterior): formedness (IF) & $-6.61 e+02$ & $3.51 e+02$ & -1.88 \\
\hline Existence (NEX): formedness (IF) & $-1.73 e+03$ & $4.79 e+02$ & -3.61 \\
\hline Session 2: F0 & $1.32 e-01$ & $2.88 e+00$ & 0.05 \\
\hline ROI (posterior): FO & $-6.65 e+00$ & $2.86 e+00$ & -2.32 \\
\hline Existence (NEX): F0 & $-1.11 e+01$ & $4.17 e+00$ & -2.67 \\
\hline Formedness (IF): F0 & $-3.17 e+01$ & $7.11 e+00$ & -4.46 \\
\hline Session 2: duration & $7.91 e+01$ & $1.85 \mathrm{e}+02$ & 0.43 \\
\hline ROI (posterior): duration & $-4.04 e+02$ & $1.84 e+02$ & -2.19 \\
\hline Existence (NEX): duration & $-5.77 e+02$ & $2.50 e+02$ & -2.31 \\
\hline Formedness (IF): duration & $-1.67 e+03$ & $3.69 e+02$ & -4.52 \\
\hline F0: duration & $-4.87 e+00$ & $3.41 e+00$ & -1.43 \\
\hline Session 2: intensity & $-1.88 e-01$ & $7.30 e-01$ & -0.26 \\
\hline ROI (posterior): intensity & $-1.67 e+00$ & $7.28 e-01$ & -2.30 \\
\hline Existence (NEX): intensity & $-2.74 e+00$ & $1.05 e+00$ & -2.62 \\
\hline Formedness (IF): intensity & $-7.81 e+00$ & $1.66 e+00$ & -4.70 \\
\hline F0: intensity & $-2.62 e-02$ & $1.34 \mathrm{e}-02$ & -1.96 \\
\hline Duration : intensity & $-1.57 e+00$ & $8.58 e-01$ & -1.82 \\
\hline Session 2: ROI (posterior): existence (NEX) & $3.90 e+02$ & $3.06 e+02$ & 1.27 \\
\hline Session 2: ROI (posterior): formedness (IF) & $6.43 e+02$ & $4.93 e+02$ & 1.30 \\
\hline Session 2: existence (NEX): formedness (IF) & $9.42 e+02$ & $4.76 e+02$ & 1.98 \\
\hline ROI (posterior): existence (NEX): formedness (IF) & $7.43 e+02$ & $4.62 e+02$ & 1.61 \\
\hline Session 2: ROI (posterior): F0 & $7.24 \mathrm{e}+00$ & $3.99 e+00$ & 1.82 \\
\hline Session 2: existence (NEX): F0 & $2.22 e+00$ & $4.38 e+00$ & 0.51 \\
\hline ROI (posterior): existence (NEX): F0 & $7.36 e+00$ & $4.30 e+00$ & 1.71 \\
\hline
\end{tabular}


Table A2. Continued.

\begin{tabular}{|c|c|c|c|}
\hline & Estimate & Std. error & $t$ \\
\hline Session 2: formedness (IF): F0 & $8.30 \mathrm{e}+00$ & $7.19 e+00$ & 1.16 \\
\hline ROI (posterior): formedness (IF): F0 & $1.27 e+01$ & $7.07 e+00$ & 1.80 \\
\hline Existence (NEX): formedness (IF): F0 & $3.48 e+01$ & $9.49 e+00$ & 3.66 \\
\hline Session 2: ROI (posterior): duration & $4.63 e+02$ & $2.56 e+02$ & 1.80 \\
\hline Session 2: existence (NEX): duration & $1.57 e+02$ & $2.62 e+02$ & 0.60 \\
\hline ROI (posterior): existence (NEX): duration & $4.20 e+02$ & $2.57 e+02$ & 1.63 \\
\hline Session 2: formedness (IF): duration & $2.72 e+02$ & $3.77 e+02$ & 0.72 \\
\hline ROI (posterior): formedness (IF): duration & $6.82 e+02$ & $3.72 e+02$ & 1.83 \\
\hline Existence (NEX): formedness (IF): duration & $1.85 e+03$ & $5.00 e+02$ & 3.69 \\
\hline Session 2: F0: duration & $-2.31 e+00$ & $3.64 e+00$ & -0.63 \\
\hline ROI (posterior): F0: duration & $7.65 e+00$ & $3.62 e+00$ & 2.11 \\
\hline Existence (NEX): F0: duration & $1.10 e+01$ & $4.91 e+00$ & 2.24 \\
\hline Formedness (IF): F0: duration & $3.25 e+01$ & $7.43 e+00$ & 4.38 \\
\hline Session 2: ROI (posterior): intensity & $1.84 \mathrm{e}+00$ & $1.01 e+00$ & 1.82 \\
\hline Session 2: existence (NEX): intensity & $9.41 e-01$ & $1.09 e+00$ & 0.86 \\
\hline ROI (posterior): existence (NEX): intensity & $1.74 \mathrm{e}+00$ & $1.07 e+00$ & 1.63 \\
\hline Session 2: formedness (IF): intensity & $2.19 e+00$ & $1.68 \mathrm{e}+00$ & 1.30 \\
\hline ROI (posterior): formedness (IF): intensity & $3.14 e+00$ & $1.66 \mathrm{e}+00$ & 1.89 \\
\hline Existence (NEX): formedness (IF): intensity & $8.34 e+00$ & $2.23 e+00$ & 3.74 \\
\hline Session 2: F0: intensity & $1.06 \mathrm{e}-03$ & $1.43 e-02$ & 0.07 \\
\hline ROI (posterior): FO: intensity & $3.15 e-02$ & $1.42 e-02$ & 2.21 \\
\hline Existence (NEX): F0: intensity & $5.37 e-02$ & $2.06 e-02$ & 2.61 \\
\hline Formedness (IF): F0: intensity & $1.54 e-01$ & $3.35 e-02$ & 4.59 \\
\hline Session 2: duration: intensity & $-2.63 e-01$ & $9.14 \mathrm{e}-01$ & -0.29 \\
\hline ROI (posterior): duration: intensity & $1.91 e+00$ & $9.10 e-01$ & 2.10 \\
\hline Existence (NEX): duration: intensity & $2.79 e+00$ & $1.23 e+00$ & 2.26 \\
\hline Formedness (IF): duration: intensity & $8.05 e+00$ & $1.74 e+00$ & 4.62 \\
\hline F0: duration: intensity & $2.47 e-02$ & $1.69 e-02$ & 1.47 \\
\hline Session 2: ROI (posterior): existence (NEX): formedness (IF) & $-7.94 e+02$ & $6.51 e+02$ & -1.22 \\
\hline Session 2: ROI (posterior): existence (NEX): F0 & $-7.34 \mathrm{e}+00$ & $6.05 e+00$ & -1.21 \\
\hline Session 2: ROI (posterior): formedness (IF): F0 & $-1.21 e+01$ & $9.93 e+00$ & -1.21 \\
\hline Session 2: existence (NEX): formedness (IF): F0 & $-1.71 e+01$ & $9.44 e+00$ & -1.81 \\
\hline ROI (posterior): existence (NEX): formedness (IF): F0 & $-1.44 e+01$ & $9.19 e+00$ & -1.57 \\
\hline Session 2: ROI (posterior): existence (NEX): duration & $-4.68 e+02$ & $3.62 e+02$ & -1.30 \\
\hline Session 2: ROI (posterior): formedness (IF): duration & $-6.83 e+02$ & $5.22 \mathrm{e}+02$ & -1.31 \\
\hline Session 2: existence (NEX): formedness (IF): duration & $-8.84 e+02$ & $5.03 e+02$ & -1.76 \\
\hline ROI (posterior): existence (NEX): formedness (IF): duration & $-7.61 e+02$ & $4.91 e+02$ & -1.55 \\
\hline Session 2: ROI (posterior): FO: duration & $-8.79 e+00$ & $5.04 e+00$ & -1.74 \\
\hline Session 2: existence (NEX): F0: duration & $-1.20 \mathrm{e}+00$ & $5.17 e+00$ & -0.23 \\
\hline ROI (posterior): existence (NEX): FO: duration & $-8.33 e+00$ & $5.09 e+00$ & -1.64 \\
\hline Session 2: formedness (IF): F0: duration & $-5.57 e+00$ & $7.59 e+00$ & -0.73 \\
\hline ROI (posterior): formedness (IF): F0: duration & $-1.32 \mathrm{e}+01$ & $7.50 e+00$ & -1.76 \\
\hline Existence (NEX): formedness (IF): F0: duration & $-3.68 e+01$ & $9.91 e+00$ & -3.71 \\
\hline Session 2: ROI (posterior): existence (NEX): intensity & $-1.94 e+00$ & $1.50 \mathrm{e}+00$ & -1.29 \\
\hline Session 2: ROI (posterior): formedness (IF): intensity & $-3.10 e+00$ & $2.33 e+00$ & -1.33 \\
\hline Session 2: existence (NEX): formedness (IF): intensity & $-4.59 e+00$ & $2.21 e+00$ & -2.08 \\
\hline ROI (posterior): existence (NEX): formedness (IF): intensity & $-3.49 e+00$ & $2.15 e+00$ & -1.62 \\
\hline Session 2: ROI (posterior): F0: intensity & $-3.49 e-02$ & $1.98 \mathrm{e}-02$ & -1.76 \\
\hline Session 2: existence (NEX): F0: intensity & $-1.07 e-02$ & $2.15 e-02$ & -0.50 \\
\hline ROI (posterior): existence (NEX): FO: intensity & $-3.45 e-02$ & $2.11 e-02$ & -1.63 \\
\hline Session 2: formedness (IF): F0: intensity & $-4.51 e-02$ & $3.39 e-02$ & -1.33 \\
\hline ROI (posterior): formedness (IF): F0: intensity & $-6.05 e-02$ & $3.34 \mathrm{e}-02$ & -1.81 \\
\hline Existence (NEX): formedness (IF): F0: intensity & $-1.68 e-01$ & $4.42 e-02$ & -3.80 \\
\hline Session 2: ROI (posterior): duration: intensity & $-2.24 e+00$ & $1.27 e+00$ & -1.77 \\
\hline Session 2: existence (NEX): duration: intensity & $-8.04 \mathrm{e}-01$ & $1.29 e+00$ & -0.63 \\
\hline ROI (posterior): existence (NEX): duration: intensity & $-1.97 e+00$ & $1.26 \mathrm{e}+00$ & -1.56 \\
\hline Session 2: formedness (IF): duration: intensity & $-1.59 e+00$ & $1.78 \mathrm{e}+00$ & -0.89 \\
\hline ROI (posterior): formedness (IF): duration: intensity & $-3.24 e+00$ & $1.76 \mathrm{e}+00$ & -1.84 \\
\hline Existence (NEX): formedness (IF): duration: intensity & $-8.89 e+00$ & $2.33 e+00$ & -3.81 \\
\hline Session 2: F0: duration: intensity & $9.02 e-03$ & $1.80 \mathrm{e}-02$ & 0.50 \\
\hline ROI (posterior): FO: duration: intensity & $-3.61 e-02$ & $1.79 e-02$ & -2.02 \\
\hline Existence (NEX): F0: duration: intensity & $-5.34 e-02$ & $2.42 e-02$ & -2.21 \\
\hline Formedness (IF): F0: duration: intensity & $-1.57 e-01$ & $3.51 e-02$ & -4.49 \\
\hline Session 2: ROI (posterior): existence (NEX): formedness (IF): F0 & $1.47 \mathrm{e}+01$ & $1.29 \mathrm{e}+01$ & 1.13 \\
\hline Session 2: ROI (posterior): existence (NEX): formedness (IF): duration & $8.03 e+02$ & $6.92 e+02$ & 1.16 \\
\hline Session 2: ROI (posterior): existence (NEX): F0: duration & $8.80 \mathrm{e}+00$ & $7.15 e+00$ & 1.23 \\
\hline Session 2: ROI (posterior): formedness (IF): F0: duration & $1.28 \mathrm{e}+01$ & $1.05 e+01$ & 1.21 \\
\hline Session 2: existence (NEX): formedness (IF): F0: duration & $1.55 e+01$ & $9.99 e+00$ & 1.55 \\
\hline ROI (posterior): existence (NEX): formedness (IF): F0: duration & $1.48 \mathrm{e}+01$ & $9.78 e+00$ & 1.51 \\
\hline Session 2: ROI (posterior): existence (NEX): formedness (IF): intensity & $3.86 e+00$ & $3.03 e+00$ & 1.27 \\
\hline Session 2: ROI (posterior): existence (NEX): F0: intensity & $3.64 e-02$ & $2.97 e-02$ & 1.22 \\
\hline
\end{tabular}


Table A2. Continued.

\begin{tabular}{|c|c|c|c|}
\hline & Estimate & Std. error & $t$ \\
\hline Session 2: ROI (posterior): formedness (IF): F0: intensity & $5.84 e-02$ & $4.70 e-02$ & 1.24 \\
\hline Session 2: existence (NEX): formedness (IF): F0: intensity & $8.30 e-02$ & $4.40 e-02$ & 1.89 \\
\hline ROI (posterior): existence (NEX): formedness (IF): F0: intensity & $6.76 e-02$ & $4.29 e-02$ & 1.58 \\
\hline Session 2: ROI (posterior): existence (NEX): duration: intensity & $2.33 e+00$ & $1.77 e+00$ & 1.31 \\
\hline Session 2: ROI (posterior): formedness (IF): duration: intensity & $3.31 e+00$ & $2.47 e+00$ & 1.34 \\
\hline Session 2: existence (NEX): formedness (IF): duration: intensity & $4.30 e+00$ & $2.34 e+00$ & 1.84 \\
\hline ROI (posterior): existence (NEX): formedness (IF): duration: intensity & $3.58 \mathrm{e}+00$ & $2.29 e+00$ & 1.56 \\
\hline Session 2: ROI (posterior): F0: duration: intensity & $4.23 e-02$ & $2.49 e-02$ & 1.70 \\
\hline Session 2: existence (NEX): F0: duration: intensity & $6.17 e-03$ & $2.54 \mathrm{e}-02$ & 0.24 \\
\hline ROI (posterior): existence (NEX): F0: duration: intensity & $3.91 e-02$ & $2.50 e-02$ & 1.56 \\
\hline Session 2: formedness (IF): F0: duration: intensity & $3.20 \mathrm{e}-02$ & $3.59 \mathrm{e}-02$ & 0.89 \\
\hline ROI (posterior): formedness (IF): F0: duration: intensity & $6.25 e-02$ & $3.55 e-02$ & 1.76 \\
\hline Existence (NEX): formedness (IF): F0: duration: intensity & $1.78 e-01$ & $4.63 e-02$ & 3.84 \\
\hline Session 2: ROI (posterior): existence (NEX): formedness (IF): F0: duration & $-1.47 e+01$ & $1.38 \mathrm{e}+01$ & -1.07 \\
\hline Session 2: ROI (posterior): existence (NEX): formedness (IF): F0: intensity & $-7.12 e-02$ & $6.04 e-02$ & -1.18 \\
\hline Session 2: ROI (posterior): existence (NEX): formedness (IF): duration: intensity & $-3.94 e+00$ & $3.23 e+00$ & -1.22 \\
\hline Session 2: ROI (posterior): existence (NEX): FO: duration: intensity & $-4.36 \mathrm{e}-02$ & $3.51 e-02$ & -1.24 \\
\hline Session 2: ROI (posterior): formedness (IF): F0: duration: intensity & $-6.20 e-02$ & $4.98 e-02$ & -1.24 \\
\hline Session 2: existence (NEX): formedness (IF): F0: duration: intensity & $-7.51 e-02$ & $4.66 e-02$ & -1.61 \\
\hline ROI (posterior): existence (NEX): formedness (IF): F0: duration: intensity & $-6.95 e-02$ & $4.57 e-02$ & -1.52 \\
\hline Session 2: ROI (posterior): existence (NEX): formedness (IF): F0: duration: intensity & $7.21 e-02$ & $6.44 e-02$ & 1.12 \\
\hline
\end{tabular}

